\title{
Disturbance Compensation Based Finite-Time Tracking Control of Rigid Manipulator
}

\author{
Mohamed Elamin Sahabi, ${ }^{1,2,3}$ Guipu Li, ${ }^{1,2}$ Xiangyu Wang, ${ }^{1,2}$ and Shihua $\mathrm{Li}^{1,2}$ \\ ${ }^{1}$ School of Automation, Southeast University, Nanjing 210096, China \\ ${ }^{2}$ Key Laboratory of Measurement and Control of Complex Systems of Engineering, Ministry of Education, China \\ ${ }^{3}$ Faculty of Engineering, Blue Nile University, P.O. Box 143, Ar-Rossieres, Blue Nile, Sudan
}

Correspondence should be addressed to Xiangyu Wang; w.x.y@seu.edu.cn

Received 19 October 2015; Accepted 15 December 2015

Academic Editor: Ricardo Aguilar-López

Copyright (C) 2016 Mohamed Elamin Sahabi et al. This is an open access article distributed under the Creative Commons Attribution License, which permits unrestricted use, distribution, and reproduction in any medium, provided the original work is properly cited.

\begin{abstract}
The finite-time tracking control problem of rigid manipulator system with mismatched disturbances is investigated via a composite control method. The proposed composite controller is based on finite-time disturbance observer and adding a power integrator technique. First, a finite-time disturbance observer is designed which guarantees that the disturbances can be estimated in a finite time. Then, a composite controller is developed based on adding a power integrator approach and the estimates of the disturbances. Under the proposed composite controller, the manipulator position can track the desired position in a finite time. Simulation results show the effectiveness of the proposed control scheme.
\end{abstract}

\section{Introduction}

Due to some superior capacities, such as high accuracy, high stiffness, and high load-carrying [1], manipulator systems have been widely used in robots [2], parallels [3], and mechanical systems [4]. To achieve the control goals for manipulators and improve the performances of the closedloop manipulator systems, PID control laws were given in [5$7]$, sliding mode control techniques were adopted in $[8,9]$, adaptive control laws were designed in [10-13], robust control algorithm was presented in [14], and neural network based control law was shown in [15].

From the point view of convergence rates, most of the existing results on control of rigid manipulators achieve asymptotic stability results for the closed-loop systems [515]. In other words, the system convergence rates are at best exponential. Compared with asymptotically stable systems, finite-time stable systems usually demonstrate faster convergence rates, better disturbance rejection properties, and robustness against uncertainties $[16,17]$. Due to such nice features, recently, the finite-time control techniques have gained increasing attention from researchers [16-36]. In these literatures, finite-time control results for manipulators are shown in [32-36].

Note that the aforementioned control schemes for manipulators with disturbances work in a robust way, which implies that the disturbances attenuation is at the price of sacrificing their nominal control performances. To improve this problem, a feasible way is to use feedforward-feedback composite control rather than pure feedback control to solve the control problems of manipulators with disturbances. Disturbance observer based control (DOBC) is an effective composite control method, which is composed of disturbance observer (DO) design and nominal feedback controller design [3740]. Compared with feedback control methods, DOBC has several superiorities, such as faster rejection of disturbances and recovery of the nominal performances. Due to such nice features, DOBC approaches have been adopted in [41-43] to reject disturbances for manipulator systems. However, the DOBC methods in these papers for the control of manipulators still have several aspects to be improved. On one hand, in the existing literatures, most DOBC techniques are only available for systems with matched disturbances: namely, the disturbances enter the system in the same channel with 
control inputs. On the other hand, the estimated disturbances converge to the real disturbances as time goes to infinity. To reject disturbances in a shorter time, a disturbance observer which provides a faster convergence rate is desired. The sliding mode differentiator in $[44,45]$ is such an observer and it has been utilized by our research group to solve the control problem of manipulators with matched disturbances in [46]. In practice, there are mismatched disturbances in the manipulators. However, up to now, there are still no finite-time control results through DOBC methods for manipulators in the presence of mismatched disturbances.

In this paper, a composite control scheme is developed for manipulator systems with mismatched disturbances. The composite control algorithm is designed based on finite-time disturbance observer (FTDO) and adding a power integrator control method. Under the proposed composite controller, in the presence of mismatched disturbances, the manipulator position can track the desired position in a finite time.

The remainder of this paper is organized as follows. Section 2 presents useful definitions and lemmas. The manipulator system model is given in Section 3. The control design is presented in Section 4. Section 5 shows the simulation results and the conclusions of this paper are drawn in Section 6.

\section{Preliminaries}

Consider the following nonlinear autonomous system:

$$
\dot{x}=f(x), \quad x \subseteq \mathbb{R}^{n}, f(0)=0,
$$

where $f: D \rightarrow \mathbb{R}^{n}$ satisfies the locally Lipschitz continuous condition. Under this condition, the definition of finite-time stability can be described as follows.

Definition 1 (finite-time stability $[16,18]$ ). The equilibrium $x=0$ of system (1) is finite-time convergent if there are an open neighbourhood $U$ of the origin and a function $T_{x}$ : $U \backslash\{0\} \rightarrow(0, \infty)$, such that every solution trajectory $x\left(t, x_{0}\right)$ of system (1) starting from the initial point $x_{0} \in U \backslash\{0\}$ is welldefined and unique in forward time for $t \in\left[0, T_{x}\left(x_{0}\right)\right)$, and $\lim _{t \rightarrow T_{x}\left(x_{0}\right)} x\left(t, x_{0}\right)=0$. Here $T_{x}\left(x_{0}\right)$ is called the convergence time (with respect to the initial state $x_{0}$ ). The equilibrium of system (1) is finite-time stable if it is Lyapunov stable and finite-time convergent. If $U=D=\mathbb{R}^{n}$, the origin is a globally finite-time stable equilibrium.

Definition 2 (homogeneity [18]). $V(x): \mathbb{R}^{n} \rightarrow \mathbb{R}$ is a continuous function. $V(x)$ is homogeneous of degree $k>0$ with respect to the dilation $\left(r_{1}, \ldots, r_{n}\right)$, if

$$
V\left(\varepsilon^{r_{1}} x_{1}, \ldots, \varepsilon^{r_{n}} x_{n}\right)=\varepsilon^{k} V(x), \quad \forall \varepsilon \in \mathbb{R}, x \in \mathbb{R}^{n} .
$$

Let $\left(r_{1}, \ldots, r_{n}\right) \in \mathbb{R}^{n}$ with $r_{i}>0, i=1, \ldots, n$, and let $f(x): \mathbb{R}^{n} \rightarrow \mathbb{R}$ be a continuous vector field. $f(x)$ is said to be homogeneous of degree $k \in \mathbb{R}$ with respect to the dilation $\left(r_{1}, \ldots, r_{n}\right)$, if for any given $\varepsilon>0$,

$$
\begin{aligned}
f_{i}\left(\varepsilon^{r_{1}} x_{1}, \ldots, \varepsilon^{r_{n}} x_{n}\right)=\varepsilon^{k+r_{i}} f_{i}(x), & \\
& i=1, \ldots, n, \forall x \in \mathbb{R}^{n},
\end{aligned}
$$

where $k \geq-\max \left\{r_{i}, i=1, \ldots, n\right\}$.
Lemma 3 (see $[16,18]$ ). Considering system (1), suppose that there exists a continuous function $V(x): U \rightarrow \mathbb{R}$ such that the following conditions hold.

(i) $V(x)$ is positive definite.

(ii) There exist real numbers $c>0, \alpha \in(0,1)$ and an open neighborhood $U_{0} \subset U$ of the origin such that

$$
\dot{V}(x)+c V^{\alpha}(x) \leq 0, \quad x \in U_{0} \backslash\{0\} .
$$

Then the origin is a finite-time stable equilibrium of system (1). If $U=U_{0}=\mathbb{R}^{n}$, the origin is a globally finite-time stable equilibrium of system (1).

Lemma 4 (see [20]). Let $c, d$ be positive real numbers and let $\gamma(x, y)>0$ be a real-valued function. Then,

$$
|x|^{c}|y|^{d} \leq \frac{c \gamma(x, y)|x|^{c+d}}{c+d}+\frac{d \gamma^{-c / d}(x, y)|y|^{c+d}}{c+d} .
$$

Lemma 5 (see [20]). For any real numbers $x_{i}, i=1, \ldots, n$, and $0<a \leq 1$, the following inequality holds:

$$
\left(\left|x_{1}\right|+\cdots+\left|x_{n}\right|\right)^{a} \leq\left|x_{1}\right|^{a}+\cdots+\left|x_{n}\right|^{a} .
$$

When $a=p / q \leq 1$, where $p>0$ and $q>0$ are odd integers, another inequality holds:

$$
\left|x^{a}-y^{a}\right| \leq 2^{1-a}|x-y|^{a} .
$$

\section{Problem Formulation}

The dynamics of a $n$ link rigid manipulator can be written as

$$
M(q) \ddot{q}+C(q, \dot{q}) \dot{q}+G(q)=\tau+d(t), \quad q \in \mathbb{R}^{n},
$$

where $q=\left[q_{1}, \ldots, q_{n}\right]^{T}, \dot{q}$ and $\ddot{q} \in \mathbb{R}^{n}$ denote the link position, velocity, and acceleration, respectively, $\tau=$ $\left[\tau_{1}, \ldots, \tau_{n}\right]^{T}$ is the control input vector, $M(q) \in \mathbb{R}^{n \times n}$ is the positive-definite symmetric inertia matrix, $C(q, \dot{q}) \in \mathbb{R}^{n \times n}$ denotes the Centrifugal-Coriolis matrix, $G(q) \in \mathbb{R}^{n}$ denotes the influence of gravity, and $d(t) \in \mathbb{R}^{n}$ is the bounded input disturbance vector. For simplicity, matrices $M(q), C(q, \dot{q})$, $G(q)$ are denoted by $M, C, G$ for short in the following.

Let $x_{1}=q$ and $x_{2}$ denote manipulator velocity. Consider mismatched disturbances and system parameter uncertainties: namely, $\dot{x}_{1}=x_{2}+d_{1}, M=M_{0}+\Delta M, C=C_{0}\left(x_{1}, x_{2}+d_{1}\right)+$ $\Delta C$, and $G=G_{0}+\Delta G$, where $M_{0}, C_{0}\left(x_{1}, x_{2}+d_{1}\right), G_{0}$ are the nominal values of $M, C, G$, respectively, and $\Delta M, \Delta G, \Delta C$ are the uncertain parts of $M, C, G$, respectively. Then, the dynamics of the manipulator can be written as

$$
\begin{aligned}
& \dot{x}_{1}=x_{2}+d_{1}, \\
& \dot{x}_{2}=M_{0}^{-1}\left(\tau-G_{0}\right)+d_{2},
\end{aligned}
$$

where $d_{1}(t) \in \mathbb{R}^{n}$ denotes the mismatched disturbances and $d_{2}(t)=M_{0}^{-1}\left[d-\Delta M\left(\dot{x}_{2}+\dot{d}_{1}\right)-M_{0} \dot{d}_{1}-\left(C_{0}\left(x_{1}, x_{2}+d_{1}\right)+\right.\right.$ $\left.\Delta C)\left(x_{2}+d_{1}\right)-\Delta G\right] \in \mathbb{R}^{n}$ denotes the matched lumped disturbances. 


\section{Controller Design and Stability Analysis}

Controller design is mainly composed of two parts, that is, disturbance observer design and composite controller design.

\subsection{Finite-Time Disturbance Observer Design}

Assumption 6. $d_{1}(t)$ and $d_{2}(t)$ in (9) are second-order differentiable and $\ddot{d}_{1}, \ddot{d}_{2}$ have Lipschitz constant vectors $L_{1}, L_{2} \in$ $\mathbb{R}^{n}$, respectively.

Remark 7. In practice, lots of disturbances satisfy Assumption 6, such as constant disturbances, ramp disturbances, and harmonic disturbances.

Under Assumption 6, to estimate the disturbances and their all-order derivatives, finite time disturbance observers will be designed in this subsection. Before proceeding, denote $\zeta=M_{0}^{-1}\left(\tau-G_{0}\right)=\left[\zeta_{1}, \ldots, \zeta_{n}\right]^{T}$. According to $[44,45], \mathrm{a}$ disturbance observer for the $i$ th $(i=1, \ldots, n)$ link of system (9) can be designed as

$$
\begin{aligned}
& \dot{\hat{x}}_{1, i}=x_{2, i}+v_{1, i}^{0} \text {, } \\
& v_{1, i}^{0}=-\lambda_{1, i}^{0} L_{1, i}^{1 / 3}\left|\widehat{x}_{1, i}-x_{1, i}\right|^{2 / 3} \operatorname{sgn}\left(\widehat{x}_{1, i}-x_{1, i}\right)+\widehat{d}_{1, i}, \\
& \dot{\hat{d}}_{1, i}=v_{1, i}^{1} \\
& =-\lambda_{1, i}^{1} L_{1, i}^{1 / 2}\left|\widehat{d}_{1, i}-v_{1, i}^{0}\right|^{1 / 2} \operatorname{sgn}\left(\widehat{d}_{1, i}-v_{1, i}^{0}\right)+\hat{\dot{d}}_{1, i}, \\
& \dot{\overrightarrow{\dot{d}}}_{1, i}=v_{1, i}^{2}=-\lambda_{1, i}^{2} L_{1, i} \operatorname{sgn}\left(\hat{\dot{d}}_{1, i}-v_{1, i}^{1}\right) \text {, } \\
& \dot{\hat{x}}_{2, i}=v_{2, i}^{0}+\zeta_{i} \text {, } \\
& v_{2, i}^{0}=-\lambda_{2, i}^{0} L_{2, i}^{1 / 3}\left|\widehat{x}_{2, i}-x_{2, i}\right|^{2 / 3} \operatorname{sgn}\left(\widehat{x}_{2, i}-x_{2, i}\right)+\widehat{d}_{2, i}, \\
& \dot{\hat{d}}_{2, i}=v_{2, i}^{1} \\
& =-\lambda_{2, i}^{1} L_{2, i}^{1 / 2}\left|\widehat{d}_{2, i}-v_{2, i}^{0}\right|^{1 / 2} \operatorname{sgn}\left(\widehat{d}_{2, i}-v_{2, i}^{0}\right)+\hat{\dot{d}}_{2, i}, \\
& \dot{\hat{\dot{d}}}_{2, i}=v_{2, i}^{2}=-\lambda_{2, i}^{2} L_{2, i} \operatorname{sgn}\left(\hat{\dot{d}}_{2, i}-v_{2, i}^{1}\right) \text {, }
\end{aligned}
$$

where $\lambda_{1, i}^{0}, \lambda_{1, i}^{1}, \lambda_{1, i}^{2}, \lambda_{2, i}^{0}, \lambda_{2, i}^{1}, \lambda_{2, i}^{2}$ are the observer coefficients to be designed and $\widehat{x}_{1, i}, \widehat{x}_{2, i}, \widehat{d}_{1, i}, \hat{\dot{d}}_{1, i}, \widehat{d}_{2, i}, \hat{\dot{d}}_{2, i}$ are the estimates of $x_{1, i}, x_{2, i}, d_{1, i}, \dot{d}_{1, i}, d_{2, i}, \dot{d}_{2, i}$, respectively.

By letting $w_{1, i}^{0}=\widehat{x}_{1, i}-x_{1, i}, w_{2, i}^{0}=\widehat{x}_{2, i}-x_{2, i}, w_{1, i}^{1}=\widehat{d}_{1, i}-$ $d_{1, i}, w_{2, i}^{1}=\widehat{d}_{2, i}-d_{2, i}, w_{1, i}^{2}=\hat{\dot{d}}_{1, i}-\dot{d}_{1, i}, w_{2, i}^{2}=\hat{\dot{d}}_{2, i}-\dot{d}_{2, i}$, the observation error system of the $i$ th link can be obtained as follows:

$$
\begin{aligned}
& \dot{w}_{1, i}^{0}=-\lambda_{1, i}^{0} L_{1, i}^{1 / 3}\left|w_{1, i}^{0}\right|^{2 / 3} \operatorname{sgn}\left(w_{1, i}^{0}\right)+w_{1, i}^{1}, \\
& \dot{w}_{1, i}^{1}=-\lambda_{1, i}^{1} L_{1, i}^{1 / 2}\left|w_{1, i}^{1}-\dot{w}_{1, i}^{0}\right|^{1 / 2} \operatorname{sgn}\left(w_{1, i}^{1}-\dot{w}_{1, i}^{0}\right)+w_{1, i}^{2}, \\
& \dot{w}_{1, i}^{2}=-\lambda_{1, i}^{2} L_{1, i} \operatorname{sgn}\left(w_{1, i}^{2}-\dot{w}_{1, i}^{1}\right)-\ddot{d}_{1, i},
\end{aligned}
$$

$$
\begin{aligned}
& \dot{w}_{2, i}^{0}=-\lambda_{2, i}^{0} L_{2, i}^{1 / 3}\left|w_{2, i}^{0}\right|^{2 / 3} \operatorname{sgn}\left(w_{2, i}^{0}\right)+w_{2, i}^{1}, \\
& \dot{w}_{2, i}^{1}=-\lambda_{2, i}^{1} L_{2, i}^{1 / 2}\left|w_{2, i}^{1}-\dot{w}_{2, i}^{0}\right|^{1 / 2} \operatorname{sgn}\left(w_{2, i}^{1}-\dot{w}_{2, i}^{0}\right)+w_{2, i}^{2}, \\
& \dot{w}_{2, i}^{2}=-\lambda_{2, i}^{2} L_{2, i} \operatorname{sgn}\left(w_{2, i}^{2}-\dot{w}_{2, i}^{1}\right)-\ddot{d}_{2, i} .
\end{aligned}
$$

From $[44,45]$, it can be obtained that disturbance observer error system (11) is finite-time stable, that is, there exists a finite time $t_{1, i}$ such that $w_{1, i}^{0}=0, w_{2, i}^{0}=0, w_{1, i}^{1}=0, w_{2, i}^{1}=$ $0, w_{1, i}^{2}=0, w_{2, i}^{2}=0$ for $t \geq t_{1, i}$. Then there is a finite time $t_{1}$ such that disturbance observation errors of $n$ links equal to zero while $t \geq t_{1}$.

\subsection{Finite-Time Controller Design}

Theorem 8. Assume that the desired position vector $q_{d}=$ $\left[q_{d 1}, \ldots, q_{d n}\right]^{T}$ is twice differentiable. For system (9), if Assumption 6 holds and controller $\tau$ is designed as

$$
\tau=M_{0}\left\{\ddot{q}_{d}-\hat{\dot{d}}_{1}-\widehat{d}_{2}-u\right\}+G_{0},
$$

the manipulator position $x_{1}$ will track the desired position $q_{d}$ in a finite time, where $1<r=r_{1} / r_{2}<2, r_{1}, r_{2}$ are positive odd integers, $k_{1}=\left[k_{1,1}, \ldots, k_{1, n}\right]^{T}, k_{2}=\left[k_{2,1}, \ldots, k_{2, n}\right]^{T}, k_{1, i}>0$, $k_{2, i}>\left\{\left(2^{1-1 / r} /(1+r)\right)\left[2^{3-1 / r} r /(1+r) k_{1, i}\right]^{r}+\left(1 / k_{1, i}\right)+(r /(1+\right.$ $\left.r))\left[4 /(1+r) k_{1, i}\right]^{1 / r}\right\}, i=1, \ldots, n$.

Proof. Defining the tracking errors as $e_{1}=\left[e_{1,1}, \ldots, e_{1, n}\right]^{T}=$ $q_{d}-x_{1}, e_{2}=\left[e_{2,1}, \ldots, e_{2, n}\right]^{T}=\dot{q}_{d}-x_{2}$, then the following tracking error system can be derived:

$$
\begin{aligned}
& \dot{e}_{1}=e_{2}-d_{1}, \\
& \dot{e}_{2}=\ddot{q}_{d}-M_{0}^{-1}\left(\tau-G_{0}\right)-d_{2} .
\end{aligned}
$$

Let $\widetilde{e}_{1}=e_{1}, \widetilde{e}_{2}=e_{2}-\widehat{d}_{1}$. Then system (13) can be written as

$$
\begin{aligned}
& \dot{\tilde{e}}_{1}=\widetilde{e}_{2}+\widehat{d}_{1}-d_{1}, \\
& \dot{\tilde{e}}_{2}=\ddot{q}_{d}-M_{0}^{-1}\left(\tau-G_{0}\right)-\widehat{d}_{2}-\dot{\vec{d}}_{1}+\widehat{d}_{2}-d_{2} .
\end{aligned}
$$

The stability analysis for closed-loop system (12) and (14) is shown in the following.

The stability analysis can be divided into two steps. In Step 1 , system states of (12) and (14) will not escape to infinity for $t<t_{1}$. In Step 2, system (12) and (14) is finite time stable when $t \geq t_{1}$.

Step 1. For the $i$ th $(i=1, \ldots, n)$ link, an energy function is defined as

$$
\begin{aligned}
& B_{i}\left(x_{i}, \widehat{x}_{i}, \widehat{d}_{i}, \hat{\dot{d}}_{i}, \tilde{e}_{i}\right)=\frac{1}{2}\left(x_{1, i}^{2}+x_{2, i}^{2}+\widehat{x}_{1, i}^{2}+\widehat{x}_{2, i}^{2}+\widehat{d}_{1, i}^{2}\right. \\
& \left.\quad+\widehat{d}_{2, i}^{2}+\hat{\dot{d}}_{1, i}^{2}+\hat{\dot{d}}_{2, i}^{2}+\widetilde{e}_{1, i}^{2}+\widetilde{e}_{2, i}^{2}\right) .
\end{aligned}
$$


Since $d_{1, i}$ and $d_{2, i}$ are estimated by $\widehat{d}_{1, i}$ and $\widehat{d}_{2, i}$ in finite time $t_{1}$, respectively, then $\left|d_{1, i}-\widehat{d}_{1, i}\right|$ and $\left|d_{2, i}-\widehat{d}_{2, i}\right|$ are bounded for $t<t_{1}$. Thus, there exist constants $w_{d_{1, i}}^{\max }$ and $w_{d_{2, i}}^{\max }$ such that $\left|d_{1, i}-\widehat{d}_{1, i}\right| \leq w_{d_{1, i}}^{\max },\left|d_{2, i}-\widehat{d}_{2, i}\right| \leq w_{d_{2, i}}^{\max }$, and $\left|\hat{\dot{d}}_{1, i}-\dot{\vec{d}}_{1, i}\right| \leq w_{\dot{d}_{1, i}}^{\max }$. Moreover, $x_{1, i} x_{2, i} \leq(1 / 2)\left(x_{1, i}^{2}+x_{2, i}^{2}\right),\left|x_{1, i}\right|^{\alpha}<1+\left|x_{1, i}\right|$ for any $0<\alpha<1$. Denote $K_{1, i}=k_{1, i}, K_{2, i}=(2-1 / r) 2^{1-1 / r} k_{1, i}^{1+r} k_{2, i}$. Then the following inequalities can be established:

$$
\begin{aligned}
& x_{1, i} \dot{x}_{1, i}=x_{1, i}\left(x_{2, i}+\widehat{d}_{1, i}+d_{1, i}-\widehat{d}_{1, i}\right) \leq \frac{1}{2}\left(x_{1, i}^{2}+x_{2, i}^{2}\right) \\
& +\frac{1}{2}\left(x_{1, i}^{2}+\widehat{d}_{1, i}^{2}\right)+\frac{1}{2}\left(\left(w_{d_{1, i}}^{\max }\right)^{2}+x_{1, i}^{2}\right), \\
& x_{2, i} \dot{x}_{2, i}=x_{2, i}\left(\ddot{q}_{d i}+K_{2, i}\left(\widetilde{e}_{2, i}^{r}+K_{1, i}^{r} \widetilde{e}_{1, i}\right)^{2 / r-1}-\hat{\dot{d}}_{1, i}\right. \\
& \left.+d_{2, i}-\widehat{d}_{2, i}\right) \\
& \leq \frac{1}{2}\left(\left(\ddot{q}_{d i}+K_{2, i}+K_{2, i} K_{1, i}^{2-r}+w_{d_{2, i}}^{\max }\right)^{2}+x_{2, i}^{2}\right)+\frac{1}{2} \\
& \cdot K_{2, i}\left(x_{2, i}^{2}+\tilde{e}_{2, i}^{2}\right)+\frac{1}{2} K_{2, i} K_{1, i}^{2-r}\left(x_{2, i}^{2}+\widetilde{e}_{1, i}^{2}\right) \\
& +\frac{1}{2}\left(x_{2, i}^{2}+\hat{\dot{d}}_{1, i}^{2}\right) \text {, } \\
& \hat{x}_{1, i} \dot{\bar{x}}_{1, i}=\widehat{x}_{1, i}\left(x_{2, i}\right. \\
& \left.-\lambda_{1, i}^{0} L_{1, i}^{1 / 3}\left|\widehat{x}_{1, i}-x_{1, i}\right|^{2 / 3} \operatorname{sgn}\left(\widehat{x}_{1, i}-x_{1, i}\right)+\widehat{d}_{1, i}\right) \\
& \leq \frac{1}{2}\left(\hat{x}_{1, i}^{2}+x_{2, i}^{2}\right)+\lambda_{1, i}^{0} L_{1, i}^{1 / 3} \hat{x}_{1, i}^{2}+\frac{1}{2} \lambda_{1, i}^{0} L_{1, i}^{1 / 3}\left(\widehat{x}_{1, i}^{2}\right. \\
& \left.+x_{1, i}^{2}\right)+\frac{1}{2} \lambda_{1, i}^{0} L_{1, i}^{1 / 3}\left(\widehat{x}_{1, i}^{2}+\widehat{d}_{1, i}^{2}\right)+\frac{1}{2}\left(\left(\lambda_{1, i}^{0} L_{1, i}^{1 / 3}\right)^{2}\right. \\
& \left.+\widehat{x}_{1, i}^{2}\right) \text {, }
\end{aligned}
$$$$
\hat{x}_{2, i} \dot{\hat{x}}_{2, i}=\widehat{x}_{2, i}\left(-\lambda_{2, i}^{0} L_{2, i}^{1 / 3}\left|\hat{x}_{2, i}-x_{2, i}\right|^{2 / 3} \operatorname{sgn}\left(\widehat{x}_{2, i}-x_{2, i}\right)\right.
$$$$
\left.+\ddot{q}_{d i}+K_{2, i}\left(\widetilde{e}_{2, i}^{r}+K_{1, i}^{r} \widetilde{e}_{1, i}\right)^{2 / r-1}-\hat{\dot{d}}_{1, i}\right)
$$$$
\leq \lambda_{2, i}^{0} L_{2, i}^{1 / 3}\left(\widehat{x}_{2, i}^{2}+\frac{1}{2}\left(\widehat{x}_{2, i}^{2}+x_{2, i}^{2}\right)\right)+\frac{1}{2}\left(\widehat{x}_{2, i}^{2}+\hat{\dot{d}}_{1, i}^{2}\right)
$$$$
+\frac{1}{2} K_{2, i}\left(\widehat{x}_{2, i}^{2}+\widetilde{e}_{2, i}^{2}\right)+\frac{1}{2} K_{2, i} K_{1, i}^{2-r}\left(\widehat{x}_{2, i}^{2}+\widetilde{e}_{1, i}^{2}\right)
$$$$
+\frac{1}{2}\left(\left(\ddot{q}_{d i}+\lambda_{2, i}^{0} L_{2, i}^{1 / 3}+K_{2, i}+K_{2, i} K_{1, i}^{2-r}\right)^{2}+\widehat{x}_{2, i}^{2}\right),
$$$$
\widehat{d}_{1, i} \dot{\hat{d}}_{1, i}=\widehat{d}_{1, i}\left(-\lambda_{1, i}^{1} L_{1, i}^{1 / 2}\left|\widehat{d}_{1, i}-v_{1, i}^{0}\right|^{1 / 2} \operatorname{sgn}\left(\widehat{d}_{1, i}-v_{1, i}^{0}\right)\right.
$$$$
\left.+\hat{\dot{d}}_{1, i}\right) \leq 2 \lambda_{1, i}^{1} L_{1, i}^{1 / 2} \widehat{d}_{1, i}^{2}+\frac{1}{2} \lambda_{1, i}^{1} L_{1, i}^{1 / 2} \lambda_{1, i}^{0} L_{1, i}^{1 / 3}\left(2 \widehat{d}_{1, i}^{2}\right.
$$$$
\left.+\widehat{x}_{1, i}^{2}+x_{1, i}^{2}\right)+\frac{1}{2}\left(\widehat{d}_{1, i}^{2}+\hat{\dot{d}}_{1, i}^{2}\right)
$$

$$
\begin{aligned}
& +\frac{1}{2}\left(\left(\lambda_{1, i}^{1} L_{1, i}^{1 / 2}+\lambda_{1, i}^{1} L_{1, i}^{1 / 2} \lambda_{1, i}^{0} L_{1, i}^{1 / 3}\right)^{2}+\widehat{d}_{1, i}^{2}\right), \\
& \widehat{d}_{2, i} \dot{\hat{d}}_{2, i}=\widehat{d}_{2, i}\left(-\lambda_{2, i}^{1} L_{2, i}^{1 / 2}\left|\hat{d}_{2, i}-v_{2, i}^{0}\right|^{1 / 2} \operatorname{sgn}\left(\hat{d}_{2, i}-v_{2, i}^{0}\right)\right. \\
& \left.+\hat{\dot{d}}_{2, i}\right) \leq 2 \lambda_{2, i}^{1} L_{2, i}^{1 / 2} \widehat{d}_{2, i}^{2}+\frac{1}{2} \lambda_{2, i}^{1} L_{2, i}^{1 / 2} \lambda_{2, i}^{0} L_{2, i}^{1 / 3}\left(2 \widehat{d}_{2, i}^{2}\right. \\
& \left.+\widehat{x}_{2, i}^{2}+x_{2, i}^{2}\right)+\frac{1}{2}\left(\widehat{d}_{2, i}^{2}+\hat{\dot{d}}_{2, i}^{2}\right) \\
& +\frac{1}{2}\left(\left(\lambda_{2, i}^{1} L_{2, i}^{1 / 2}+\lambda_{2, i}^{1} L_{2, i}^{1 / 2} \lambda_{2, i}^{0} L_{2, i}^{1 / 3}\right)^{2}+\widehat{d}_{2, i}^{2}\right), \\
& \hat{\dot{d}}_{1, i} \dot{\dot{\dot{d}}}_{1, i}=\hat{\dot{d}}_{1, i}\left(-\lambda_{1, i}^{2} L_{1, i} \operatorname{sgn}\left(\hat{\dot{d}}_{1, i}-v_{1, i}^{1}\right)\right) \\
& \leq \frac{1}{2}\left(\left(\lambda_{1, i}^{2} L_{1, i}\right)^{2}+\hat{\dot{d}}_{1, i}^{2}\right), \\
& \hat{\dot{d}}_{2, i} \dot{\dot{\dot{d}}}_{2, i}=\hat{\dot{d}}_{2, i}\left(-\lambda_{2, i}^{2} L_{2, i} \operatorname{sgn}\left(\hat{\dot{d}}_{2, i}-v_{2, i}^{1}\right)\right) \\
& \leq \frac{1}{2}\left(\left(\lambda_{2, i}^{2} L_{2, i}\right)^{2}+\hat{\dot{d}}_{2, i}^{2}\right) \\
& \widetilde{e}_{1, i} \dot{\tilde{e}}_{1, i}=\widetilde{e}_{1, i}\left(\widetilde{e}_{2, i}+\widehat{d}_{1, i}-d_{1, i}\right) \leq \frac{1}{2}\left(\widetilde{e}_{1, i}^{2}+\widetilde{e}_{2, i}^{2}\right) \\
& +\frac{1}{2}\left(\left(w_{d_{1, i}}^{\max }\right)^{2}+\widetilde{e}_{1, i}^{2}\right) \\
& \widetilde{e}_{2, i} \dot{\tilde{e}}_{2, i}=\widetilde{e}_{2, i}\left(-K_{2, i}\left(\widetilde{e}_{2, i}^{r}+K_{1, i}^{r} \widetilde{e}_{1, i}\right)^{2 / r-1}+\hat{\dot{d}}_{1, i}-\dot{\vec{d}}_{1, i}\right. \\
& \left.+\widehat{d}_{2, i}-d_{2, i}\right) \leq K_{2, i} \widetilde{e}_{2, i}^{2}+\frac{1}{2} K_{1, i}^{2-r} K_{2, i}\left(\widetilde{e}_{1, i}^{2}+\widetilde{e}_{2, i}^{2}\right) \\
& +\frac{1}{2}\left(\left(K_{2, i}+K_{1, i}^{2-r} K_{2, i}+w_{d_{2, i}}^{\max }+w_{\dot{d}_{1, i}}^{\max }\right)^{2}+\widetilde{e}_{2, i}^{2}\right) .
\end{aligned}
$$

Taking the first derivative of $B_{i}\left(x_{i}, \widehat{x}_{i}, \widehat{d}_{i}, \hat{\dot{d}}_{i}, \widetilde{e}_{i}\right)$ yields

$$
\dot{B}_{i} \leq K_{i} B_{i}+C_{i},
$$

where

$$
\begin{aligned}
K_{i} & =\max \left\{\frac{3}{2}+\frac{1}{2} \lambda_{1, i}^{0} L_{1, i}^{1 / 3}+\frac{1}{2} \lambda_{1, i}^{1} L_{1, i}^{1 / 2} \lambda_{1, i}^{0} L_{1, i}^{1 / 3}, 2+\frac{1}{2}\right. \\
& \cdot K_{2, i}+\frac{1}{2} K_{2, i} K_{1, i}^{2-r}+\frac{1}{2} \lambda_{2, i}^{0} L_{2, i}^{1 / 3}+\frac{1}{2} \lambda_{2, i}^{1} L_{2, i}^{1 / 2} \lambda_{2, i}^{0} L_{2, i}^{1 / 3} \\
& 1+2 \lambda_{1, i}^{0} L_{1, i}^{1 / 3}+\frac{1}{2} \lambda_{1, i}^{1} 1_{1, i}^{1 / 2} \lambda_{1, i}^{0} L_{1, i}^{1 / 3}, 1+\frac{1}{2} K_{2, i}+\frac{1}{2} \\
\cdot & K_{2, i} K_{1, i}^{2-r}+\frac{3}{2} \lambda_{2, i}^{0} L_{2, i}^{1 / 3}+\frac{1}{2} \lambda_{2, i}^{1} L_{2, i}^{1 / 2} \lambda_{2, i}^{0} L_{2, i}^{1 / 3} \frac{3}{2}+\frac{1}{2} \\
\cdot & \lambda_{1, i}^{0} L_{1, i}^{1 / 3}+2 \lambda_{1, i}^{1} L_{1, i}^{1 / 2}+\lambda_{1, i}^{1} L_{1, i}^{1 / 2} \lambda_{1, i}^{0} L_{1, i}^{1 / 3}, 1 \\
& +2 \lambda_{2, i}^{1} L_{2, i}^{1 / 2}+\lambda_{2, i}^{1} L_{2, i}^{1 / 2} \lambda_{2, i}^{0} L_{2, i}^{1 / 3}, 2,1,1+\frac{3}{2} K_{2, i} K_{1, i}^{2-r}, 1 \\
& \left.+2 K_{2, i}+\frac{1}{2} K_{2, i} K_{1, i}^{2-r}\right\},
\end{aligned}
$$




$$
\begin{aligned}
C= & \max \left\{\frac{1}{2}\left(w_{d_{1, i}}^{\max }\right)^{2},\right. \\
& \frac{1}{2}\left(\ddot{q}_{d i}+K_{2, i}+K_{2, i} K_{1, i}^{2-r}+w_{d_{2, i}}^{\max }\right)^{2}, \frac{1}{2}\left(\lambda_{1, i}^{0} L_{1, i}^{1 / 3}\right)^{2}, \\
& \frac{1}{2}\left(\ddot{q}_{d i}+\lambda_{2, i}^{0} L_{2, i}^{1 / 3}+K_{2, i}+K_{2, i} K_{1, i}^{2-r}\right)^{2}, \\
& \frac{1}{2}\left(\lambda_{1, i}^{1} L_{1, i}^{1 / 2}+\lambda_{1, i}^{1} L_{1, i}^{1 / 2} \lambda_{1, i}^{0} L_{1, i}^{1 / 3}\right)^{2}, \\
& \frac{1}{2}\left(\lambda_{2, i}^{1} L_{2, i}^{1 / 2}+\lambda_{2, i}^{1} L_{2, i}^{1 / 2} \lambda_{2, i}^{0} L_{2, i}^{1 / 3}\right)^{2}, \frac{1}{2}\left(\lambda_{1, i}^{2} L_{1, i}\right)^{2}, \\
& \left.\frac{1}{2}\left(\lambda_{2, i}^{2} L_{2, i}\right)^{2}, \frac{1}{2}\left(K_{2, i}+K_{1, i}^{2-r} K_{2, i}+w_{d_{2, i}}^{\max }+w_{\dot{d}_{1, i}}^{\max }\right)^{2}\right\} .
\end{aligned}
$$

Solving inequality (17), it can be obtained that $B_{i}\left(x_{i}, \widehat{x}_{i}, \widehat{d}_{i}, \hat{\dot{d}}_{i}\right.$, $\left.\tilde{e}_{i}\right) \leq\left(B_{i}\left(x_{i}(0), \hat{x}_{i}(0), \hat{d}_{i}(0), \hat{\dot{d}}_{i}(0), \widetilde{e}_{i}(0)\right)+C_{i} / K_{i}\right) e^{K_{i} t}-C_{i} / K_{i}$. Hence, system states $\widetilde{e}_{1, i}, \widetilde{e}_{2, i}$ are bounded when $t<t_{1}$.

Step 2. It will be shown that closed-loop system (12) and (14) is finite time stable when $t \geq t_{1}$. Before proceeding, denote $u=\left[u_{1}, \ldots, u_{n}\right]^{T}=\ddot{q}_{d}-M_{0}^{-1}\left(\tau-G_{0}\right)-\widehat{d}_{2}-\hat{\dot{d}}_{1}$. While $t \geq t_{1}$, system (14) reduces to

$$
\begin{aligned}
& \dot{\tilde{e}}_{1}=\widetilde{e}_{2}, \\
& \dot{\tilde{e}}_{2}=u .
\end{aligned}
$$

For the $i$ th $(i=1, \ldots, n)$ link, choose a Lyapunov function $V_{1}\left(\widetilde{e}_{1, i}\right)=\widetilde{e}_{1, i}^{2} / 2$. Taking the derivative of $V_{1}\left(\widetilde{e}_{1, i}\right)$ yields $\dot{V}_{1}\left(\widetilde{e}_{1, i}\right)=\widetilde{e}_{1, i} \widetilde{e}_{2, i}=\widetilde{e}_{1, i}\left(\widetilde{e}_{2, i}-\widetilde{e}_{2, i}^{*}\right)+\widetilde{e}_{1, i} \widetilde{e}_{2, i}^{*}$, where $\widetilde{e}_{2, i}^{*}$ is a virtual control law which can be defined as $\widetilde{e}_{2, i}^{*}=-k_{1, i} \widetilde{e}_{1, i}^{1 / r}$. Then the following inequality can be derived:

$$
\dot{V}_{1}\left(\widetilde{e}_{1, i}\right) \leq-k_{1, i} \tilde{e}_{1, i}^{1+1 / r}+\widetilde{e}_{1, i}\left(\widetilde{e}_{2, i}-\widetilde{e}_{2, i}^{*}\right)
$$

Define $\xi_{2, i}=\widetilde{e}_{2, i}^{r}-\tilde{e}_{2, i}^{* r}$. Choose the Lyapunov function:

$$
V_{2}\left(\widetilde{e}_{1, i}, \widetilde{e}_{2, i}\right)=V_{1}\left(\widetilde{e}_{1, i}\right)+\frac{\int_{\tilde{e}_{2, i}^{*}}^{\widetilde{e}_{2, i}}\left(s^{r}-\widetilde{e}_{2, i}^{* r}\right)^{2-1 / r} \mathrm{~d} s}{(2-1 / r) 2^{1-1 / r} k_{1, i}^{1+r}} .
$$

By noting that $\partial \widetilde{e}_{2, i}^{* r} / \partial \widetilde{e}_{1, i}=-k_{1, i}^{r}$, the derivative of $V_{2}\left(\widetilde{e}_{1, i}, \widetilde{e}_{2, i}\right)$ along system (19) satisfies

$$
\begin{aligned}
\dot{V}_{2}\left(\widetilde{e}_{1, i}, \widetilde{e}_{2, i}\right) \leq & -k_{1, i} \tilde{e}_{1, i}^{1+1 / r}+\widetilde{e}_{1, i}\left(\widetilde{e}_{2, i}-\tilde{e}_{2, i}^{*}\right) \\
& +\frac{\widetilde{e}_{2, i}}{2^{1-1 / r} k_{1, i}} \int_{\widetilde{e}_{2, i}^{*}}^{\tilde{e}_{2, i}}\left(s^{r}-\widetilde{e}_{2, i}^{* r}\right)^{1-1 / r} \mathrm{~d} s \\
& +\frac{\xi_{2, i}^{2-1 / r} u_{i}}{(2-1 / r) 2^{1-1 / r} k_{1, i}^{1+r}} .
\end{aligned}
$$

From Lemma 5, it can be obtained that $\left|\widetilde{e}_{2, i}-\widetilde{e}_{2, i}^{*}\right| \leq$ $2^{1-1 / r}\left|\xi_{2, i}\right|^{1 / r}$. It follows from (22) that

$$
\begin{aligned}
\dot{V}_{2}\left(\widetilde{e}_{1, i}, \widetilde{e}_{2, i}\right) \leq & -k_{1, i} \tilde{e}_{1, i}^{1+1 / r}+2^{1-1 / r}\left|\widetilde{e}_{1, i}\right|\left|\xi_{2, i}\right|^{1 / r} \\
& +\frac{\left|\widetilde{e}_{2, i}\right|\left|\xi_{2, i}\right|}{k_{1, i}}+\frac{\xi_{2, i}^{2-1 / r} u_{i}}{(2-1 / r) 2^{1-1 / r} k_{1, i}^{1+r}} .
\end{aligned}
$$

From Lemma 4, it can be obtained that

$$
2^{1-1 / r}\left|\widetilde{e}_{1, i}\right|\left|\xi_{2, i}\right|^{1 / r} \leq \frac{2^{1-1 / r} r}{1+r} \gamma \tilde{e}_{1, i}^{1+1 / r}+\frac{2^{1-1 / r}}{1+r} \gamma^{-r} \xi_{2, i}^{1+1 / r},
$$

where $\gamma$ can be any positive constant. Letting $\left(2^{1-1 / r} /(1+\right.$ $r)) \gamma=k_{1, i} / 4$ yields

$$
\begin{aligned}
2^{1-1 / r}\left|\widetilde{e}_{1, i}\right|\left|\xi_{2, i}\right|^{1 / r} \leq & \frac{k_{1, i}}{4} \tilde{e}_{1, i}^{1+1 / r} \\
& +\frac{2^{1-1 / r}}{1+r}\left[\frac{2^{3-1 / r} r}{(1+r) k_{1, i}}\right]^{r} \xi_{2, i}^{1+1 / r} .
\end{aligned}
$$

Note that $\tilde{e}_{2, i}=\left(\xi_{2, i}+\tilde{e}_{2, i}^{* r}\right)^{1 / r}$. Using Lemma 5, it can be obtained that $\left|\widetilde{e}_{2, i}\right| \leq\left|\xi_{2, i}\right|^{1 / r}+\left|\widetilde{e}_{2, i}^{* r}\right|=\left|\xi_{2, i}\right|^{1 / r}+k_{1, i}\left|\widetilde{e}_{1, i}\right|^{1 / r}$; thus, $\left|\widetilde{e}_{2, i} \xi_{2, i}\right| / k_{1, i} \leq \xi_{2, i}^{1+1 / r} / k_{1, i}+\left|\widetilde{e}_{1, i}\right|^{1 / r}\left|\xi_{2, i}\right|$. It follows from Lemma 4 that

$$
\left|\tilde{e}_{1, i}\right|^{1 / r}\left|\xi_{2, i}\right| \leq \frac{\gamma}{1+r} \tilde{e}_{1, i}^{1+1 / r}+\frac{r}{1+r} \gamma^{-1 / r} \xi_{2, i}^{1+1 / r}
$$

where $\gamma$ can be any positive constant. By letting $\gamma /(1+r)=$ $k_{1, i} / 4$, it follows that

$$
\begin{aligned}
\frac{\left|\widetilde{e}_{2, i} \xi_{2, i}\right|}{k_{1, i}} \leq & \frac{\xi_{2, i}^{1+1 / r}}{k_{1, i}}+\frac{k_{1, i}}{4} \widetilde{e}_{1, i}^{1+1 / r} \\
& +\frac{r}{1+r}\left[\frac{4}{(1+r) k_{1, i}}\right]^{1 / r} \xi_{2, i}^{1+1 / r} .
\end{aligned}
$$

Substituting (24) and (27) into (28) yields

$$
\begin{aligned}
\dot{V}_{2}\left(\widetilde{e}_{1, i}, \widetilde{e}_{2, i}\right) \leq & -\frac{k_{1, i}}{2} \widetilde{e}_{1, i}^{1+1 / r}+\delta \xi_{2, i}^{1+1 / r} \\
& +\frac{\xi_{2, i}^{2-1 / r} u_{i}}{(2-1 / r) 2^{1-1 / r} k_{1, i}^{1+r}}
\end{aligned}
$$

where

$$
\begin{aligned}
\delta= & \left\{\frac{2^{1-1 / r}}{1+r}\left[\frac{2^{3-1 / r} r}{(1+r) k_{1, i}}\right]^{r}+\frac{1}{k_{1, i}}\right. \\
& \left.+\frac{r}{1+r}\left[\frac{4}{(1+r) k_{1, i}}\right]^{1 / r}\right\} .
\end{aligned}
$$

Controller $u_{i}$ can be designed as

$$
u_{i}=-\left(2-\frac{1}{r}\right) 2^{1-1 / r} k_{1, i}^{1+r} k_{2, i}\left(\widetilde{e}_{2, i}^{r}+k_{1, i}^{r} \widetilde{e}_{1, i}\right)^{2 / r-1},
$$


where $k_{2, i}>\delta$. Substituting (30) into (28) yields

$$
\dot{V}_{2}\left(\widetilde{e}_{1, i}, \widetilde{e}_{2, i}\right) \leq-\frac{k_{1, i}}{2} \widetilde{e}_{1, i}^{1+1 / r}-k_{3, i} \xi_{2, i}^{1+1 / r}
$$

where $k_{3, i}=k_{2, i}-\delta>0$. By using Lemma 5, it follows that $\dot{V}_{2}+c V_{2}^{(1+r) / 2 r} \leq-k_{i}\left(\widetilde{e}_{1, i}^{1+1 / r}+\xi_{2, i}^{1+1 / r}\right) / 2 \leq 0$, where $k_{i}=\max \left\{k_{1, i} / 2-c(1 / 2)^{(1+r) / 2 r}, k_{3, i}-c\left(1 /(2-1 / r) k_{1, i}^{1+r}\right)^{(1+r) / 2 r}\right\}$. According to Lemma 3, it can be concluded that closed-loop system (19) and (30) is finite-time stable. Then system (12) and (14) is finite-time stable; that is, manipulator position $q$ can track the desired trajectory $q_{d}$ in a finite time. This completes the proof.

Remark 9. Under the condition without disturbance observer, with adding a power integrator method, a controller for manipulator system (9) can be designed as

$$
\tau=M_{0}\left(\ddot{q}_{d}-\bar{u}\right)+G_{0}
$$

where $\bar{u}=\left[\bar{u}_{1}, \ldots, \bar{u}_{2}\right]^{T}, \bar{u}_{i}=-(2-1 / \bar{r}) 2^{1-1 / \bar{r}} \bar{k}_{1, i}^{1+\bar{r}} \bar{k}_{2, i}\left[\left(\dot{q}_{d i}-\right.\right.$ $\left.\left.x_{2, i}\right)^{\bar{r}}+\bar{k}_{1, i}^{\bar{r}}\left(q_{d i}-x_{1, i}\right)\right]^{2 / \bar{r}-1}, 1<\bar{r}=\bar{r}_{1} / \bar{r}_{2}<2, \bar{r}_{1}, \bar{r}_{2}$ are positive odd integers, $\bar{k}_{1, i}>0, \bar{k}_{2, i}>\left\{\left(2^{1-1 / \bar{r}} /(1+\bar{r})\right)\left[2^{3-1 / \bar{r}} \bar{r} /(1+\right.\right.$ $\left.\left.\bar{r}) \bar{k}_{1, i}\right]^{\bar{r}}+1 / \bar{k}_{1, i}+(\bar{r} /(1+\bar{r}))\left[4 /(1+\bar{r}) \bar{k}_{1, i}\right]^{1 / \bar{r}}\right\}, i=1, \ldots, n$. Under controller (32), manipulator position $q$ can not track the desired trajectory $q_{d}$ in any long time, which will be shown in simulations.

\section{Numerical Simulations}

Simulations are conducted on a two-link rigid robot manipulator. The cases without/with output noises are considered in the simulations. The manipulator model is shown in Figure 1. The dynamic of the manipulator is

$$
\begin{aligned}
& {\left[\begin{array}{cc}
a_{11}\left(q_{2}\right) & a_{12}\left(q_{2}\right) \\
a_{12}\left(q_{2}\right) & a_{22}
\end{array}\right]\left[\begin{array}{c}
\ddot{q}_{1} \\
\ddot{q}_{2}
\end{array}\right]} \\
& +\left[\begin{array}{c}
-\beta_{12}\left(q_{2}\right) \dot{q}_{1}^{2}-2 \beta_{12}\left(q_{2}\right) \dot{q}_{1} \dot{q}_{2} \\
\beta_{12}\left(q_{2}\right) \dot{q}_{2}^{2}
\end{array}\right] \\
& +\left[\begin{array}{c}
\gamma_{1}\left(q_{1}, q_{2}\right) g \\
\gamma_{2}\left(q_{1}, q_{2}\right) g
\end{array}\right]=\left[\begin{array}{c}
\tau_{1} \\
\tau_{2}
\end{array}\right],
\end{aligned}
$$

where

$$
\begin{aligned}
a_{11}\left(q_{2}\right)= & \left(m_{1}+m_{2}\right) l_{1}^{2}+m_{2} l_{2}^{2}+2 m_{2} l_{1} l_{2} \cos \left(q_{2}\right) \\
& +J_{1}, \\
a_{12}\left(q_{2}\right)= & m_{2} l_{2}^{2}+m_{2} l_{1} l_{2} \cos \left(q_{2}\right), \\
a_{22}= & m_{2} l_{2}^{2}+J_{2}, \\
\beta_{12}\left(q_{2}\right)= & m_{2} l_{1} l_{2} \sin \left(q_{2}\right), \\
\gamma_{1}\left(q_{1}, q_{2}\right)= & \left(m_{1}+m_{2}\right) l_{1} \cos \left(q_{2}\right) \\
& +m_{2} l_{2} \cos \left(q_{1}+q_{2}\right), \\
\gamma_{2}\left(q_{1}, q_{2}\right)= & m_{2} l_{2} \cos \left(q_{1}+q_{2}\right) .
\end{aligned}
$$

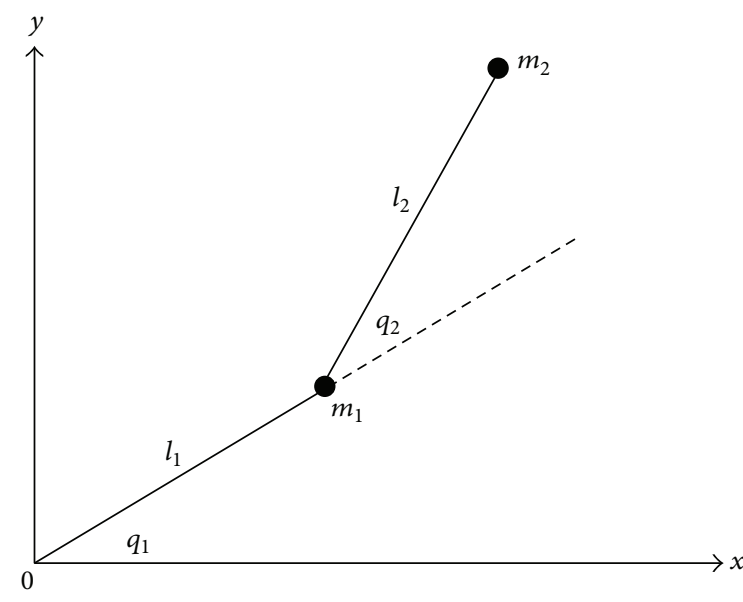

FIgURE 1: The two-link robot manipulator model.

The reference trajectories are [32]

$$
\begin{aligned}
& q_{d 1}=1.25-1.4 e^{-t}+0.35 e^{-4 t}, \quad t \geq 0 \\
& q_{d 2}=1.25+e^{-t}-0.25 e^{-4 t}, \quad t \geq 0
\end{aligned}
$$

The system parameters and disturbances are selected as $l_{1}=$ $1 \mathrm{~m}, l_{2}=0.8 \mathrm{~m}, J_{1}=5 \mathrm{kgm}, J_{2}=5 \mathrm{kgm}, m_{1}=0.4 \mathrm{~kg}, m_{2}=$ $1.2 \mathrm{~kg}, \Delta m_{1}=0.004 \mathrm{~kg}, \Delta m_{2}=0.01 \mathrm{~kg}, d_{1}=[0.03 \cos (2 t) ;$ $0.05 \cos (2 t)], d=[3 \sin (2 t) ; 5 \sin (2 t)]$.

5.1. The Case without Output Measurement Noises. To validate the effectiveness of the proposed composite control algorithm, closed-loop system performances under composite controller (12) and under finite-time controller (32) will be compared in this part.

Taking the practical input saturation into consideration, the control inputs for both controller (12) and controller (32) are limited not to exceed $60 \mathrm{Nm}$. Under these limitations, efforts have been spent to make both closed-loop systems as good as possible. For composite controller (12), the parameters are chosen as $r=15 / 13, k_{1}=[0.8 ; 0.6], k_{2}=[4.8 ; 3.5]$. The observer gains and Lipschitz constants are chosen as $\lambda_{1}^{0}=$ $[12 ; 10], \lambda_{1}^{1}=[5 ; 5], \lambda_{1}^{2}=[2 ; 2], \lambda_{2}^{0}=[10 ; 8], \lambda_{2}^{1}=[2 ; 2]$, $\lambda_{2}^{2}=[1 ; 0.5], L_{1}=[10 ; 20], L_{2}=[10 ; 20]$. For finite-time controller (32), the parameters are selected as $\bar{r}=15 / 13$, $\bar{k}_{1}=[0.8 ; 0.6], \bar{k}_{2}=[21 ; 23]$.

Simulation results are presented in Figures 2-4. Figures 2 and 3 show that observation errors of the disturbance observers converge to the origin in a finite time for both links of the manipulator. From Figure 4, it can be seen that manipulator positions can track the desired positions in a finite time under controller (12) while they cannot track the desired positions under controller (32).

5.2. The Case with Output Measurement Noises. In this part, in the presence of output measurement noises, the closedloop system performances under composite controller (12) and under finite-time controller (32) are compared to validate the effectiveness of composite controller (12). 


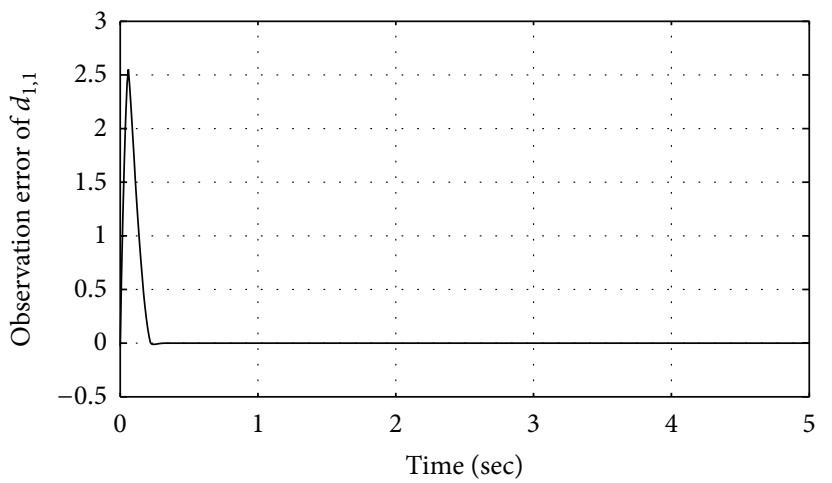

(a)

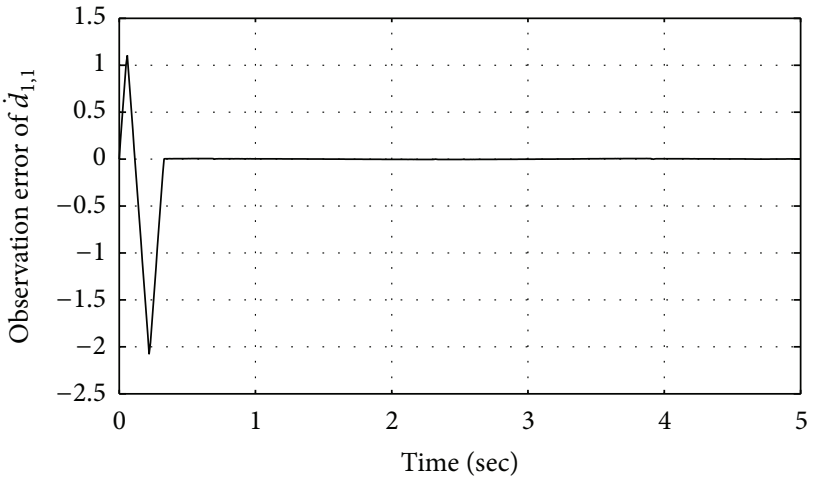

(b)

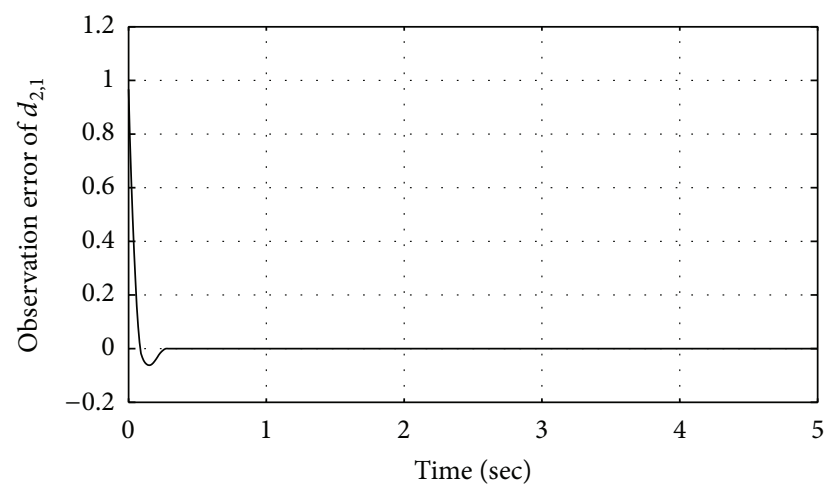

(c)

FIGURE 2: Disturbance observation errors of the 1st link. (a) Observation error of $d_{1,1}$. (b) Observation error of $\dot{d}_{1,1}$. (c) Observation error of $d_{2,1}$.

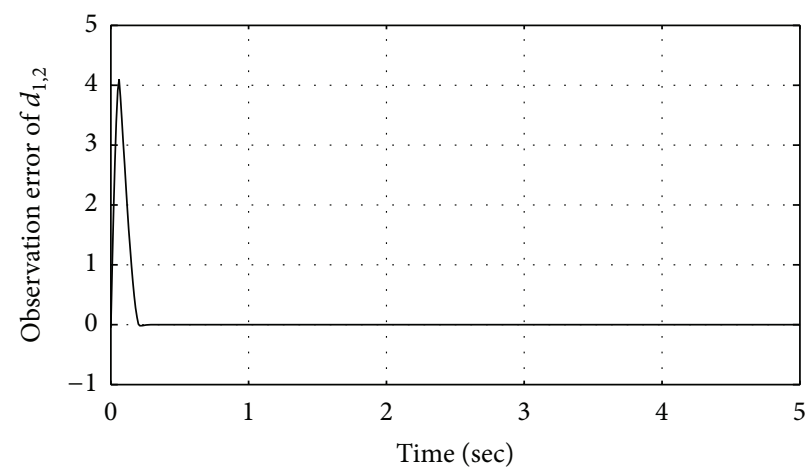

(a)

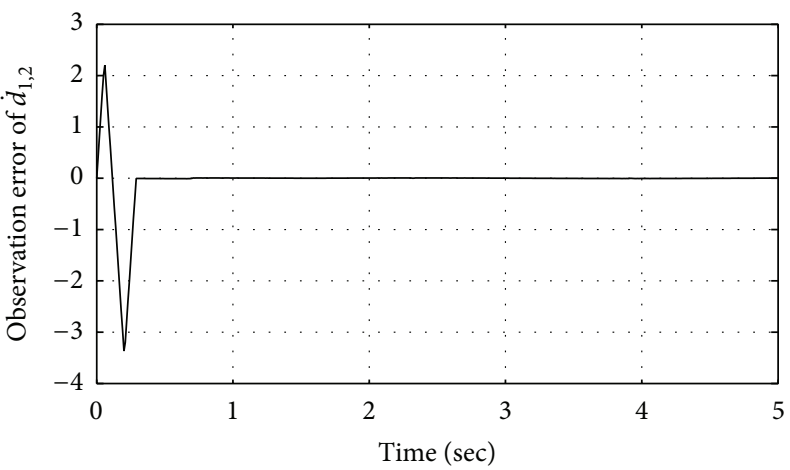

(b)

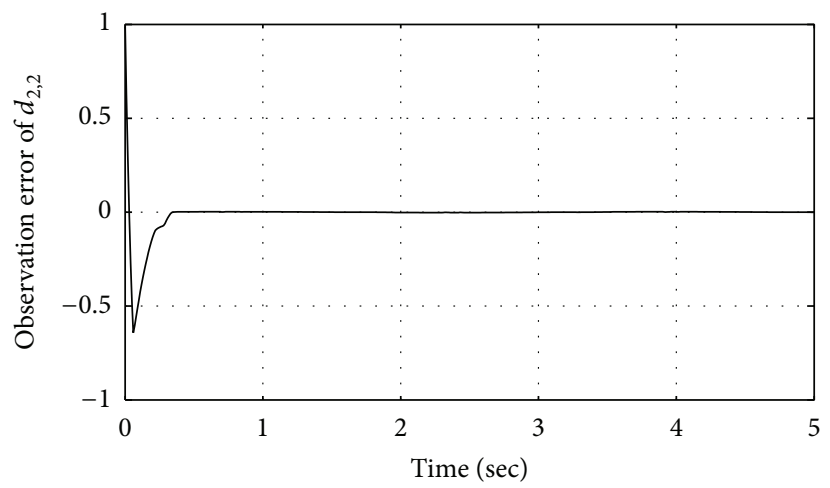

(c)

FiguRE 3: Disturbance observation errors of the 2nd link. (a) Observation error of $d_{1,2}$. (b) Observation error of $\dot{d}_{1,2}$. (c) Observation error of $d_{2,2}$. 

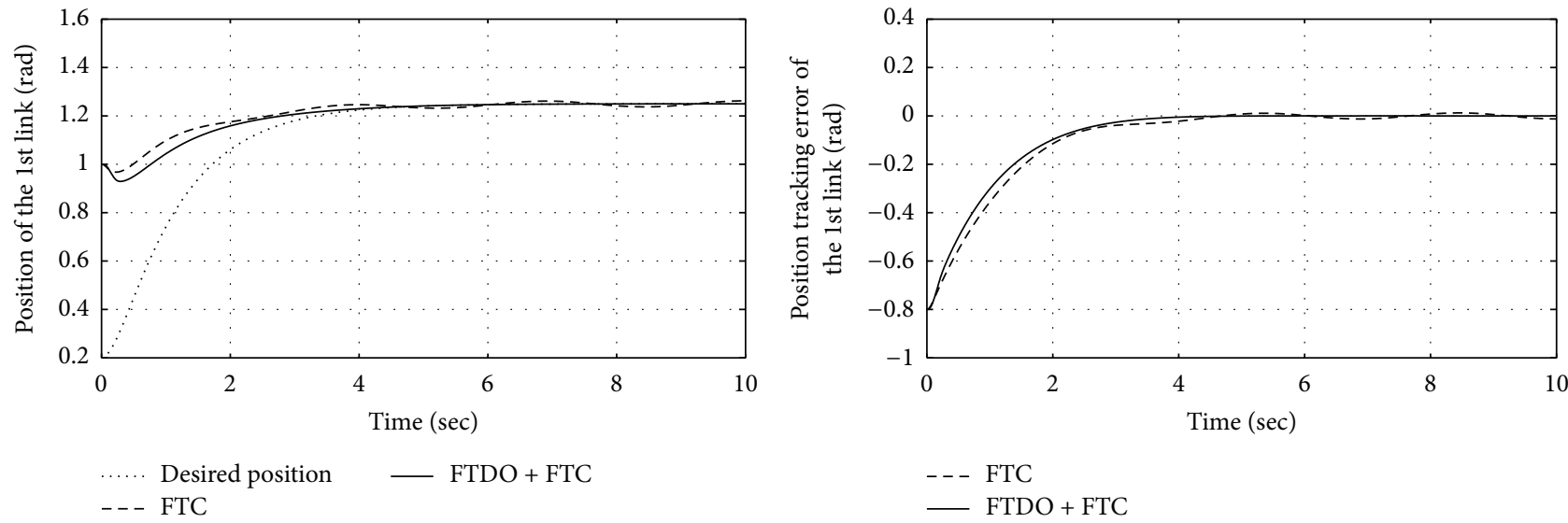

(a)

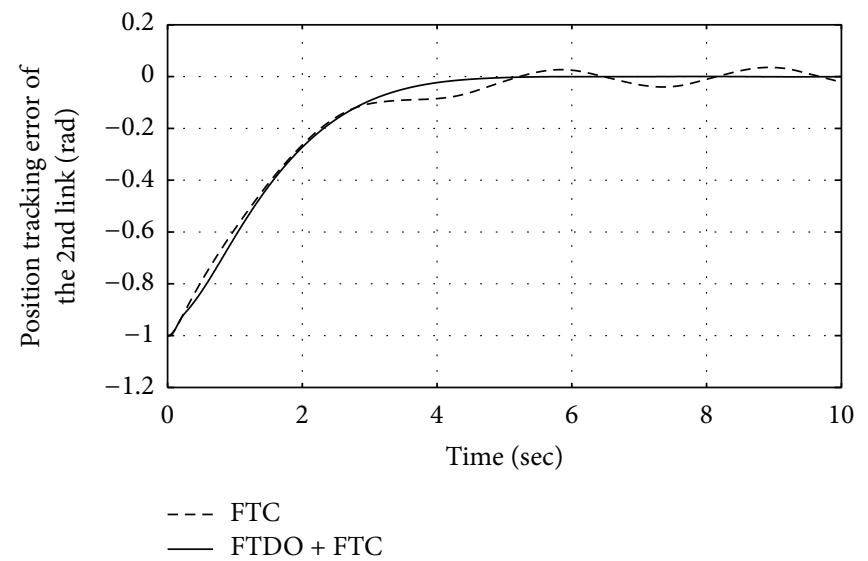

(d)

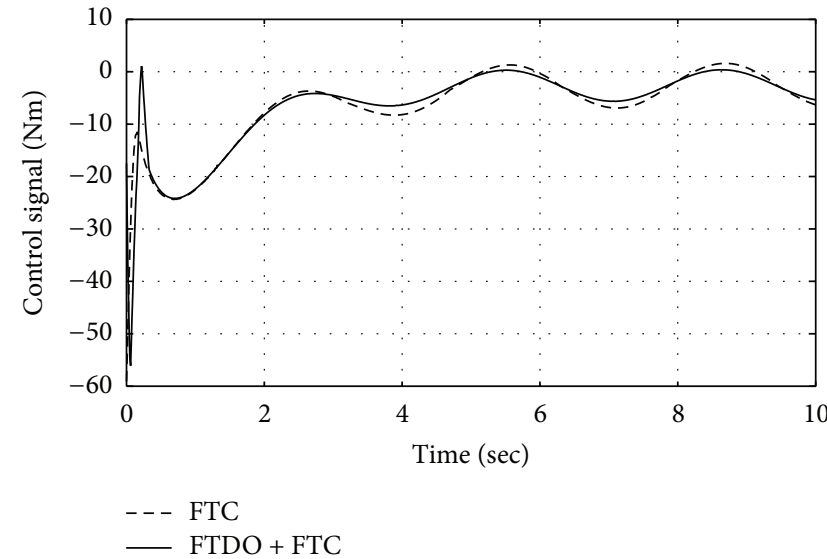

(e)

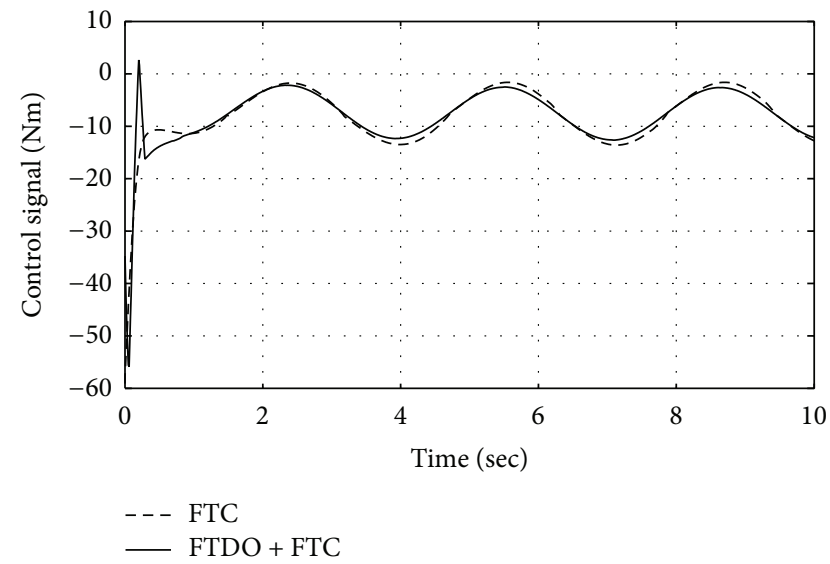

(f)

Figure 4: Response curves of system (9) under controllers (12) and (32). (a) Positions of the 1st link (rad). (b) Position tracking errors of the 1st link (rad). (c) Positions of the 2nd link (rad). (d) Position tracking errors of the 2nd link (rad). (e) Control signals for the 1st link (Nm). (f) Control signals for the 2nd link (Nm).

Generally speaking, in practice, higher-frequency measurement noises can be filtered by some filters, for example, Kalman filters. In this way, the measured states used by the controllers are usually signals with only lower-frequency noises. Hence, in simulations, only lower-frequency noises are considered and the output measurement noises are assumed to be $n=[0.01 \sin (5 t) ; 0.01 \cos (5 t)]$. In other words, the measured output is $y=x_{1}+n$. Differentiating $y$ yields $\dot{y}=x_{2}+\bar{d}_{1}$, where $\bar{d}_{1}=d_{1}+\dot{n}\left(d_{1}\right.$ is defined in system (9)). By an observer almost the same as (10) (the only difference is that the observer estimates $\bar{d}_{1}$ rather than $d_{1}$ in system (9)), the mismatched disturbance $\bar{d}_{1}$ can still be estimated. Then based on the disturbances estimates, a composite controller almost the same as (12) (the only difference is the replacement 


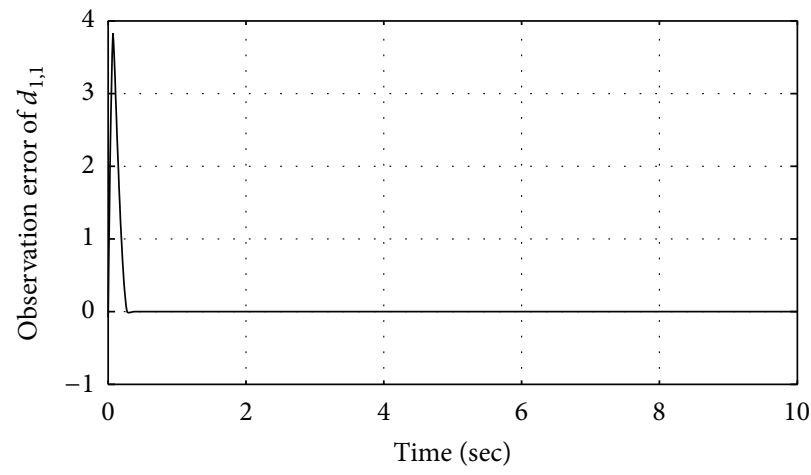

(a)

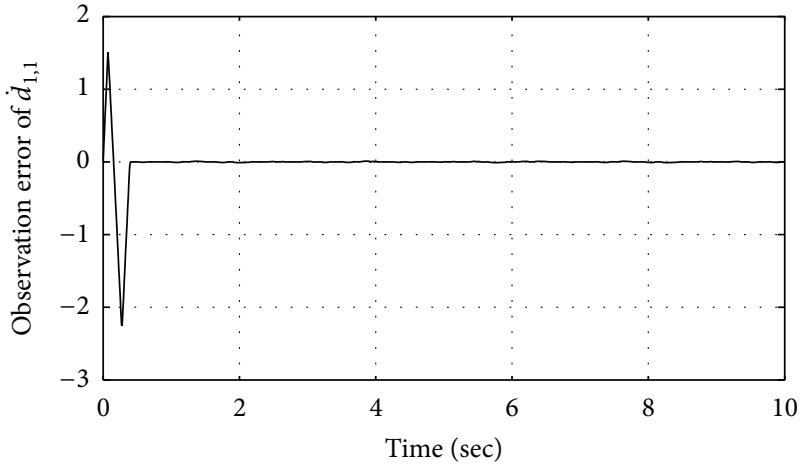

(b)

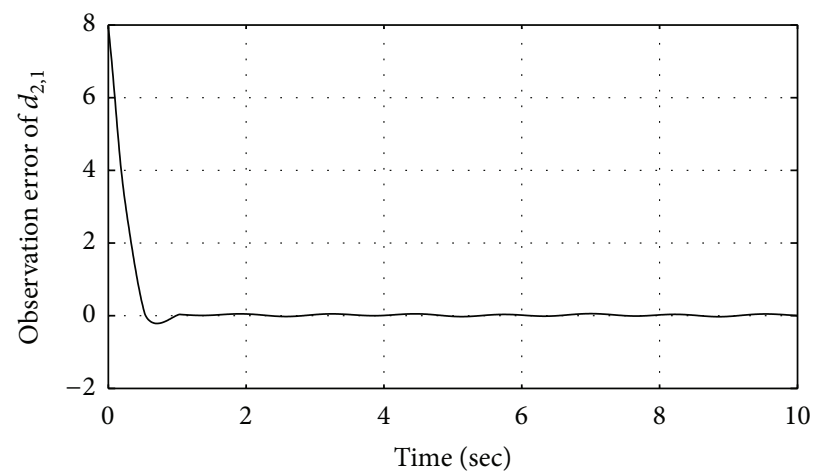

(c)

Figure 5: Disturbance observation errors of the 1st link in the presence of output measurement noises. (a) Observation error of $d_{1,1}$. (b) Observation error of $\dot{d}_{1,1}$. (c) Observation error of $d_{2,1}$.

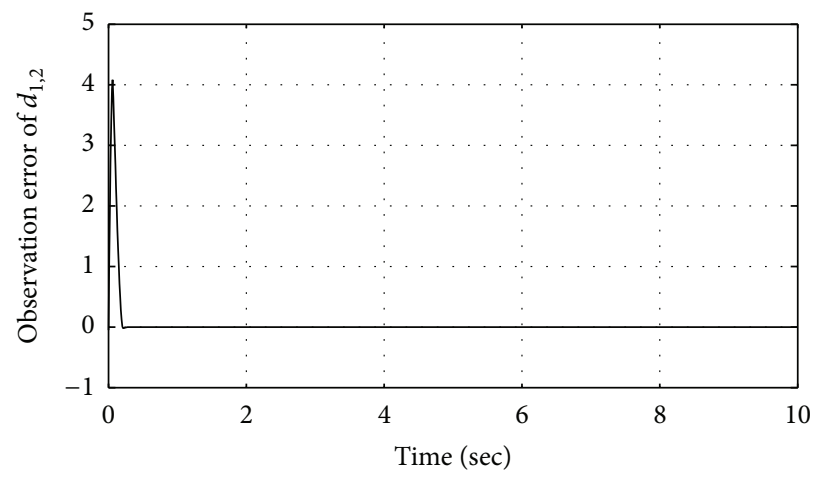

(a)

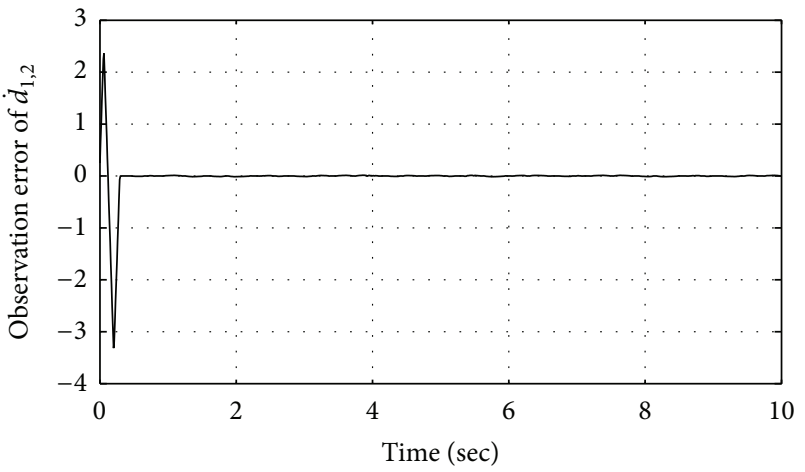

(b)

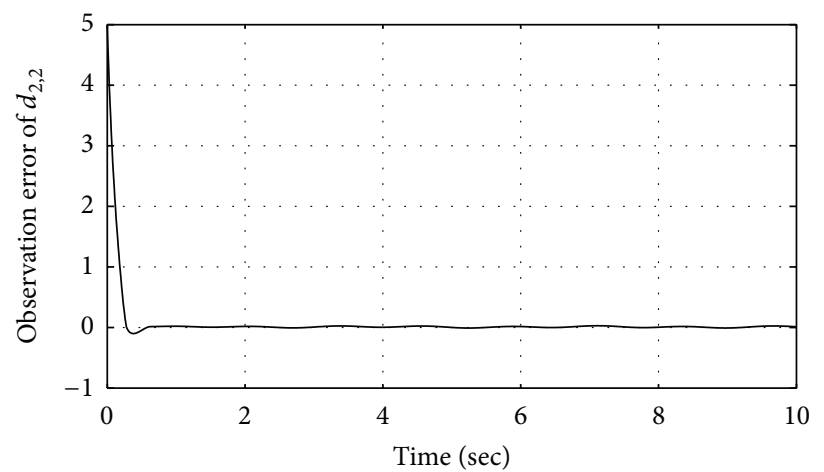

(c)

FiguRE 6: Disturbance observation errors of the 2 nd link in the presence of output measurement noises. (a) Observation error of $d_{1,2}$. (b) Observation error of $\dot{d}_{1,2}$. (c) Observation error of $d_{2,2}$. 


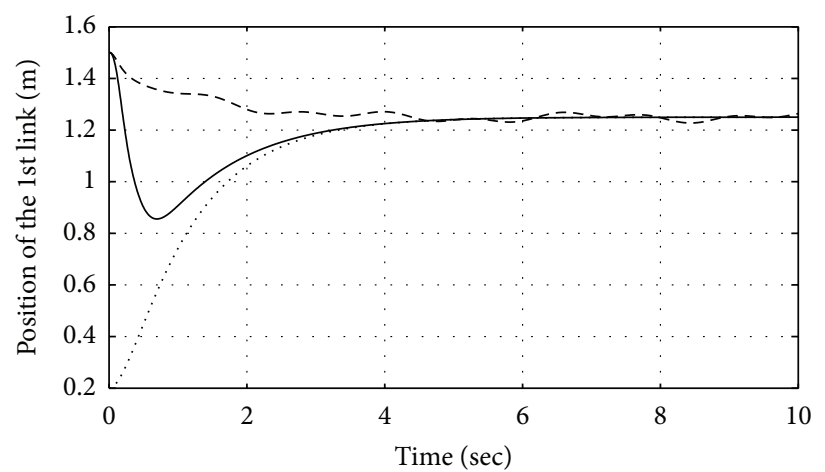

$\ldots$ D... Desired position $\quad-$ FTDO + FTC
- - FTC

(a)

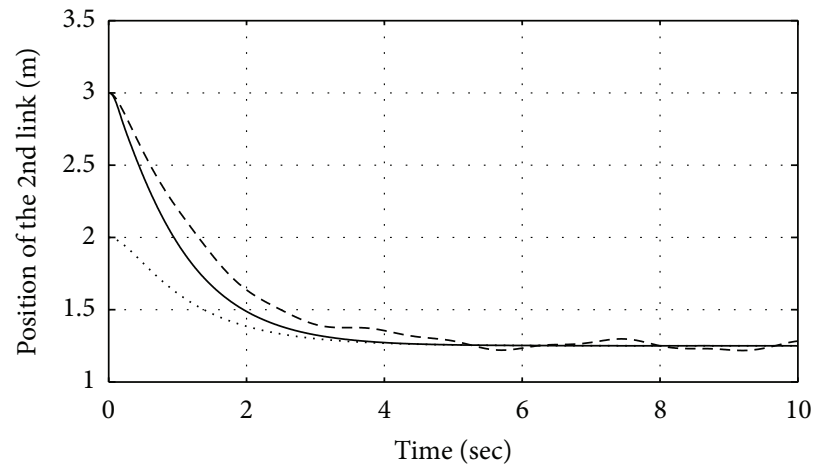

..... Desired position $\quad$ FTDO + FTC

.. - FTC

(c)

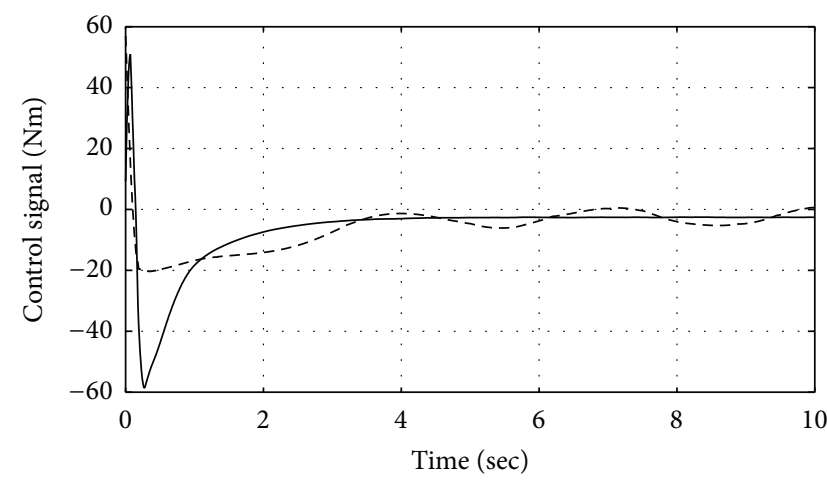

-.- FTC

- FTDO + FTC

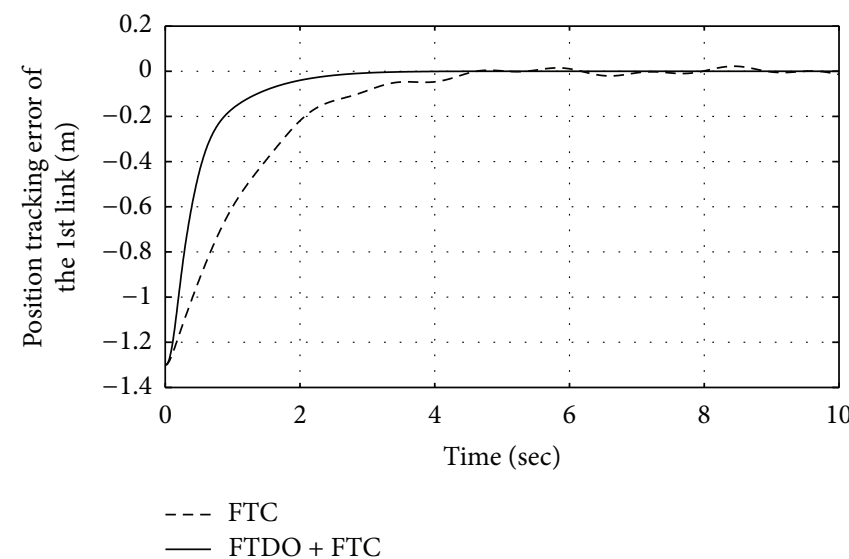

(b)

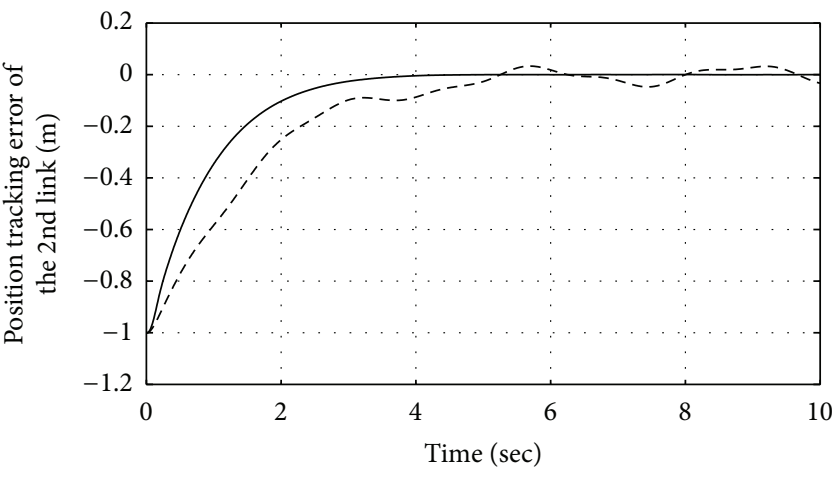

- - - FTC

- FTDO + FTC

(d)

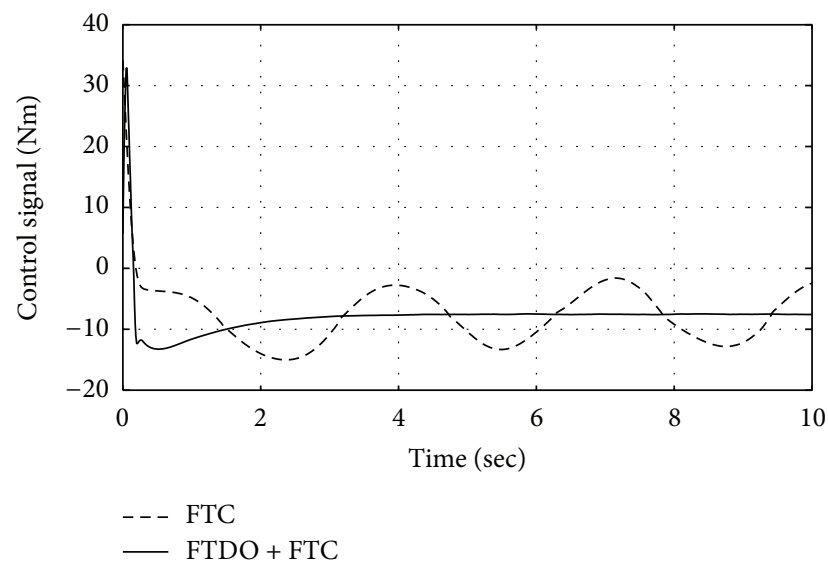

(f)

Figure 7: Response curves of system (9) under controllers (12) and (32) in the presence of output measurement noises. (a) Positions of the 1st link (rad). (b) Position tracking errors of the 1st link (rad). (c) Positions of the 2nd link (rad). (d) Position tracking errors of the 2nd link (rad). (e) Control signals for the lst link (Nm). (f) Control signals for the 2nd link (Nm).

of $x_{1}, \widehat{d}_{1}, \hat{\dot{d}}_{1}$ by $y=x_{1}+n, \widehat{\bar{d}}_{1}, \hat{\bar{d}}_{1}$, resp.) can be designed. Under the same input saturation as the case without output noises, the parameters for disturbance observer (10) and composite controller (12) are selected as $r=15 / 13, k_{1}=[0.9 ; 0.7]$, $k_{2}=[4.5 ; 3.5], \lambda_{1}^{0}=[12 ; 10], \lambda_{1}^{1}=[5 ; 5], \lambda_{1}^{2}=[2 ; 2]$, $\lambda_{2}^{0}=[10 ; 8], \lambda_{2}^{1}=[2 ; 2], \lambda_{2}^{2}=[1 ; 0.5], L_{1}=[10 ; 20]$,
$L_{2}=[10 ; 20]$. For finite-time controller (32), the parameters are selected as $\bar{r}=15 / 13, \bar{k}_{1}=[0.8 ; 0.6], \bar{k}_{2}=[18 ; 25]$.

Simulation results are given in Figures $5-7$. Figures 5 and 6 show that, even in the presence of lower-frequency noises, observer (10) still works well and provides accurate disturbances estimates in a fast way. Moreover, manipulator 
positions can still track the desired positions in a finite time under controller (12) while controller (32) fails to do this.

\section{Conclusions}

This paper has studied the position tracking control problem of rigid manipulator system with mismatched disturbances. By using adding a power integrator technique and FTDO method, a composite control scheme has been developed. The proposed control method has realized that the manipulator positions tracked the desired positions in finite time and simulations have shown the effectiveness of the proposed composite control algorithm.

\section{Conflict of Interests}

The authors declare that there is no conflict of interests regarding the publication of this paper.

\section{Acknowledgments}

This work was supported by the National Natural Science Foundation of China under Grants 61473080 and 61503078, the Natural Science Foundation of Jiangsu Province under Grant BK20150626, China Postdoctoral Science Foundation Funded Project under Grant 2015M570398, the Fundamental Research Funds for the Central Universities under Grant 2242015K40029, and the Priority Academic Program Development of Jiangsu Higher Education Institutions.

\section{References}

[1] D. Zhao, S. Li, and Q. Zhu, "A new TSMC prototype robust nonlinear task space control of a 6 DOF parallel robotic manipulator," International Journal of Control, Automation and Systems, vol. 8, no. 6, pp. 1189-1197, 2010.

[2] Y. Su, P. C. Muller, and C. Zheng, "Global asymptotic saturated PID control for robot manipulators," IEEE Transactions on Control Systems Technology, vol. 18, no. 6, pp. 1280-1288, 2010.

[3] D. Zhao, S. Li, and F. Gao, "Finite time position synchronised control for parallel manipulators using fast terminal sliding mode," International Journal of Systems Science, vol. 40, no. 8, pp. 829-843, 2009.

[4] Y. Su, "A simple global asymptotic convergent observer for uncertain mechanical systems," International Journal of Systems Science, vol. 47, no. 4, pp. 903-912, 2016.

[5] Y. Su, D. Sun, L. Ren, and J. K. Mills, "Integration of saturated PI synchronous control and PD feedback for control of parallel manipulators," IEEE Transactions on Robotics, vol. 22, no. 1, pp. 202-207, 2006.

[6] A. Zavala-Río and V. Santibáñez, "A natural saturating extension of the PD-with-desired-gravity-compensation control law for robot manipulators with bounded inputs," IEEE Transactions on Robotics, vol. 23, no. 2, pp. 386-391, 2007.

[7] J. Alvarez-Ramirez, R. Kelly, and I. Cervantes, "Semiglobal stability of saturated linear PID control for robot manipulators," Automatica, vol. 39, no. 6, pp. 989-995, 2003.

[8] S. Islam and X. P. Liu, "Robust sliding mode control for robot manipulators," IEEE Transactions on Industrial Electronics, vol. 58, no. 6, pp. 2444-2453, 2011.
[9] L. M. Capisani and A. Ferrara, "Trajectory planning and second-order sliding mode motion/interaction control for robot manipulators in unknown environments," IEEE Transactions on Industrial Electronics, vol. 59, no. 8, pp. 3189-3198, 2012.

[10] D. Braganza, W. E. Dixon, D. M. Dawson, and B. Xian, "Tracking control for rigid manipulators with kinematic and dynamic uncertainty," International Journal of Robotics and Automation, vol. 23, no. 2, pp. 117-126, 2008.

[11] E. Zergeroglu, W. Dixon, A. Behal, and D. Dawson, "Adaptive set-point control of robotic manipulators with amplitudelimited control inputs," Robotica, vol. 18, no. 2, pp. 171-181, 2000.

[12] W. E. Dixon, "Adaptive regulation of amplitude limited rigid manipulators with uncertain kinematics and dynamics," IEEE Transactions on Automatic Control, vol. 52, no. 3, pp. 488-493, 2007.

[13] M. Boukattaya, M. Jallouli, and T. Damak, “On trajectory tracking control for nonholonomic mobile manipulators with dynamic uncertainties and external torque disturbances," Robotics and Autonomous Systems, vol. 60, no. 12, pp. 1640-1647, 2012.

[14] W. Gueaieb, S. Al-Sharhan, and M. Bolic, "Robust computationally efficient control of cooperative closed-chain manipulators with uncertain dynamics," Automatica, vol. 43, no. 5, pp. 842851, 2007.

[15] L. Cui, H. Zhang, B. Chen, and Q. Zhang, "Asymptotic tracking control scheme for mechanical systems with external disturbances and friction," Neurocomputing, vol. 73, no. 7-9, pp. 1293$1302,2010$.

[16] S. P. Bhat and D. S. Bernstein, "Finite-time stability of continuous autonomous systems," SIAM Journal on Control and Optimization, vol. 38, no. 3, pp. 751-766, 2000.

[17] S. Li, H. Du, and X. Lin, "Finite-time consensus algorithm for multi-agent systems with double-integrator dynamics," Automatica, vol. 47, no. 8, pp. 1706-1712, 2011.

[18] S. P. Bhat and D. S. Bernstein, "Geometric homogeneity with applications to finite-time stability," Mathematics of Control, Signals, and Systems, vol. 17, no. 2, pp. 101-127, 2005.

[19] H. Du, S. Li, and C. Qian, "Finite-time attitude tracking control of spacecraft with application to attitude synchronization," IEEE Transactions on Automatic Control, vol. 56, no. 11, pp. 2711-2717, 2011.

[20] X. Huang, W. Lin, and B. Yang, "Global finite-time stabilization of a class of uncertain nonlinear systems," Automatica, vol. 41, no. 5, pp. 881-888, 2005.

[21] C. Qian and J. Li, "Global finite-time stabilization by output feedback for planar systems without observable linearization," IEEE Transactions on Automatic Control, vol. 50, no. 6, pp. 885890, 2005.

[22] S. Li, H. Sun, J. Yang, and X. Yu, "Continuous finite-time output regulation for disturbed systems under mismatching condition," IEEE Transactions on Automatic Control, vol. 60, no. 1, pp. 277-282, 2015.

[23] H. Du, C. Qian, S. Yang, and S. Li, "Recursive design of finitetime convergent observers for a class of time-varying nonlinear systems," Automatica, vol. 49, no. 2, pp. 601-609, 2013.

[24] X. Wang, S. Li, and P. Shi, "Distributed finite-time containment control for double-integrator multiagent systems," IEEE Transactions on Cybernetics, vol. 44, no. 9, pp. 1518-1528, 2014.

[25] X. Wang, X. Sun, S. Li, and H. Ye, "Output feedback domination approach for finite-time force control of an electrohydraulic actuator," IET Control Theory \& Applications, vol. 6, no. 7, pp. 921-934, 2012. 
[26] S. Khoo, J. Yin, Z. Man, and X. Yu, "Finite-time stabilization of stochastic nonlinear systems in strict-feedback form," Automatica, vol. 49, no. 5, pp. 1403-1410, 2013.

[27] Y. Guo and L. Chen, "Terminal sliding mode control for coordinated motion of a space rigid manipulator with external disturbance," Applied Mathematics and Mechanics, vol. 29, no. 5, pp. 583-590, 2008.

[28] Y. Feng, X. Yu, and F. Han, "On nonsingular terminal slidingmode control of nonlinear systems," Automatica, vol. 49, no. 6, pp. 1715-1722, 2013.

[29] Y. Feng, F. Han, and X. Yu, "Chattering free full-order slidingmode control," Automatica, vol. 50, no. 4, pp. 1310-1314, 2014.

[30] S. Li and X. Wang, "Finite-time consensus and collision avoidance control algorithms for multiple AUVs," Automatica, vol. 49, no. 11, pp. 3359-3367, 2013.

[31] S. Li, X. Wang, and L. Zhang, "Finite-time output feedback tracking control for autonomous underwater vehicles," IEEE Journal of Oceanic Engineering, vol. 40, no. 3, pp. 727-751, 2015.

[32] Y. Feng, X. Yu, and Z. Man, "Non-singular terminal sliding mode control of rigid manipulators," Automatica, vol. 38, no. 12, pp. 2159-2167, 2002.

[33] Y. Hong, Y. Xu, and J. Huang, "Finite-time control for robot manipulators," Systems \& Control Letters, vol. 46, no. 4, pp. $243-$ 253, 2002.

[34] X. Wang, X. Sun, S. Li, and H. Ye, "Finite-time position tracking control of rigid hydraulic manipulators based on highorder terminal sliding mode," Proceedings of the Institution of Mechanical Engineers. Part I: Journal of Systems and Control Engineering, vol. 226, no. 3, pp. 394-414, 2012.

[35] Y. Su, "Global continuous finite-time tracking of robot manipulators," International Journal of Robust and Nonlinear Control, vol. 19, no. 17, pp. 1871-1885, 2009.

[36] D. Zhao, S. Li, Q. Zhu, and F. Gao, "Robust finite-time control approach for robotic manipulators," IET Control Theory \& Applications, vol. 4, no. 1, pp. 1-15, 2010.

[37] W.-H. Chen, D. J. Ballance, P. J. Gawthrop, and J. O'Reilly, "A nonlinear disturbance observer for robotic manipulators," IEEE Transactions on Industrial Electronics, vol. 47, no. 4, pp. 932-938, 2000.

[38] L. Guo and W.-H. Chen, "Disturbance attenuation and rejection for systems with nonlinearity via DOBC approach," International Journal of Robust and Nonlinear Control, vol. 15, no. 3, pp. 109-125, 2005.

[39] S. Li, J. Yang, W. Chen, and X. Chen, Disturbance ObserverBased Control: Methods and Applications, CRC Press, Boca Raton, Fla, USA, 2014.

[40] W. Chen, J. Yang, L. Guo, and S. Li, "Disturbance observer-based control and related methods: an overview," IEEE Transactions on Industrial Electronics, 2015.

[41] C. Zhongyi, S. Fuchun, and C. Jing, "Disturbance observerbased robust control of free-floating space manipulators," IEEE Systems Journal, vol. 2, no. 1, pp. 114-119, 2008.

[42] Y. Pi and X. Wang, "Observer-based cascade control of a 6DOF parallel hydraulic manipulator in joint space coordinate," Mechatronics, vol. 20, no. 6, pp. 648-655, 2010.

[43] Z. Chu, J. Cui, and F. Sun, "Fuzzy adaptive disturbanceobserver-based robust tracking control of electrically driven free-floating space manipulator," IEEE Systems Journal, vol. 8, no. 2, pp. 343-352, 2014.

[44] A. Levant, "Higher-order sliding modes, differentiation and output-feedback control," International Journal of Control, vol. 76, no. 9-10, pp. 924-941, 2003.
[45] Y. B. Shtessel, I. A. Shkolnikov, and A. Levant, "Smooth secondorder sliding modes: missile guidance application," Automatica, vol. 43, no. 8, pp. 1470-1476, 2007.

[46] J. Su, J. Yang, and S. Li, "Finite-time disturbance rejection control for robotic manipulators based on sliding mode differentiator," in Proceedings of the 25th Chinese Control and Decision Conference (CCDC '13), pp. 3844-3849, IEEE, Guiyang, China, May 2013. 


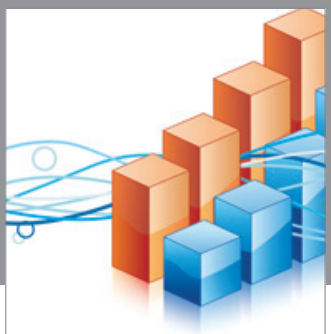

Advances in

Operations Research

vatem alat4

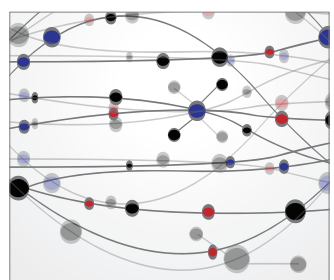

\section{The Scientific} World Journal
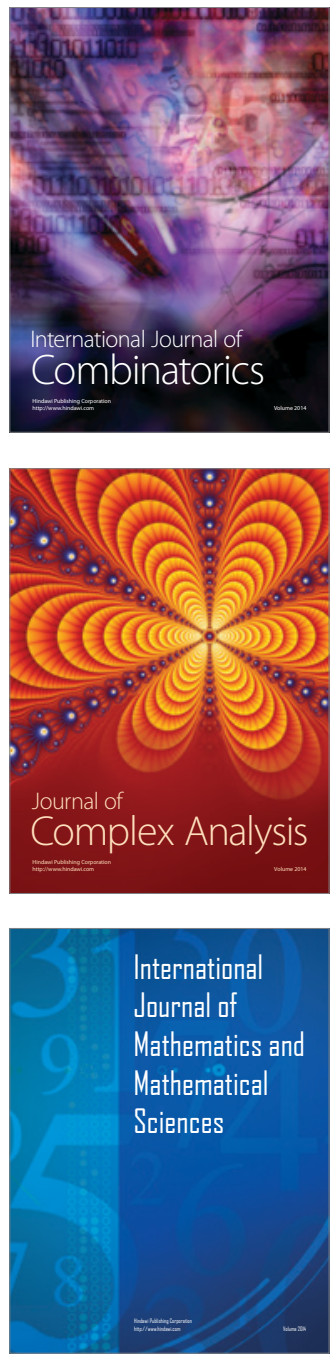
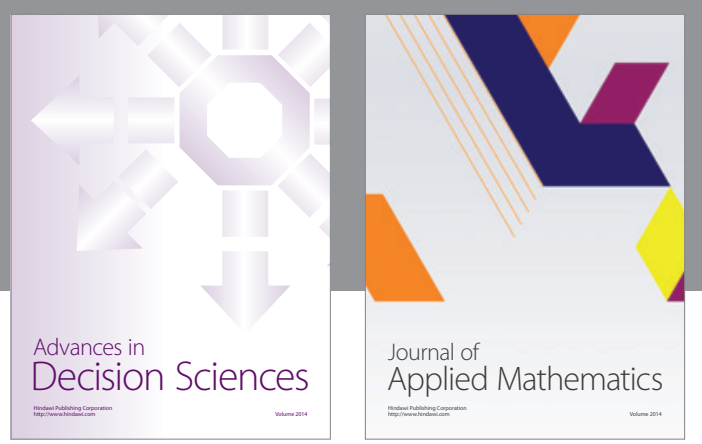

Algebra

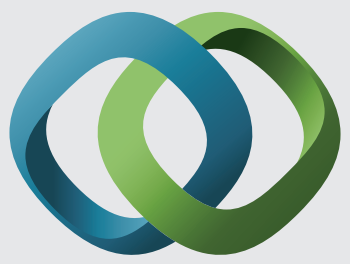

\section{Hindawi}

Submit your manuscripts at

http://www.hindawi.com
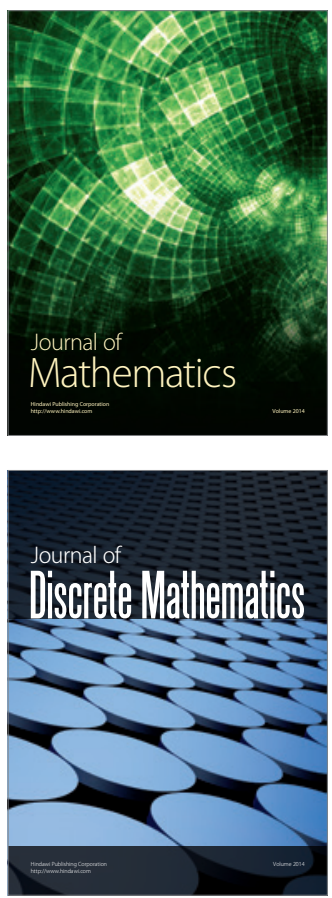

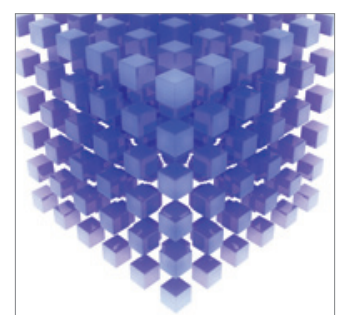

Mathematical Problems in Engineering
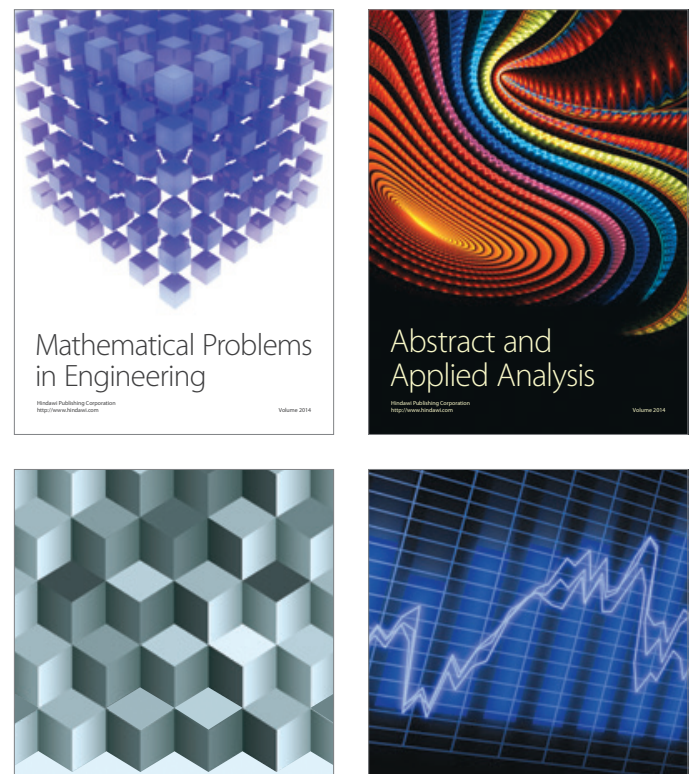

Journal of

Function Spaces

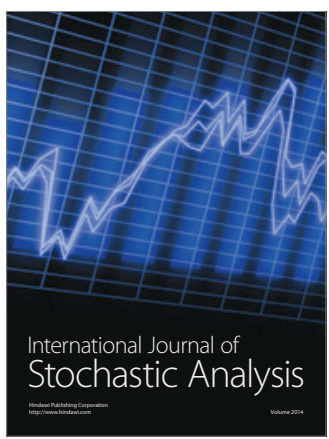

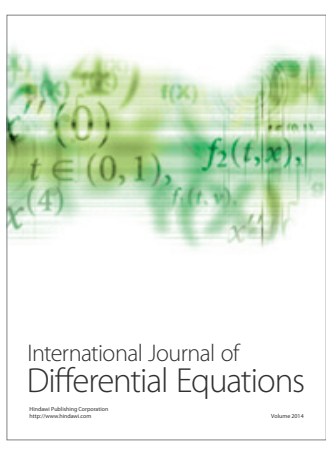
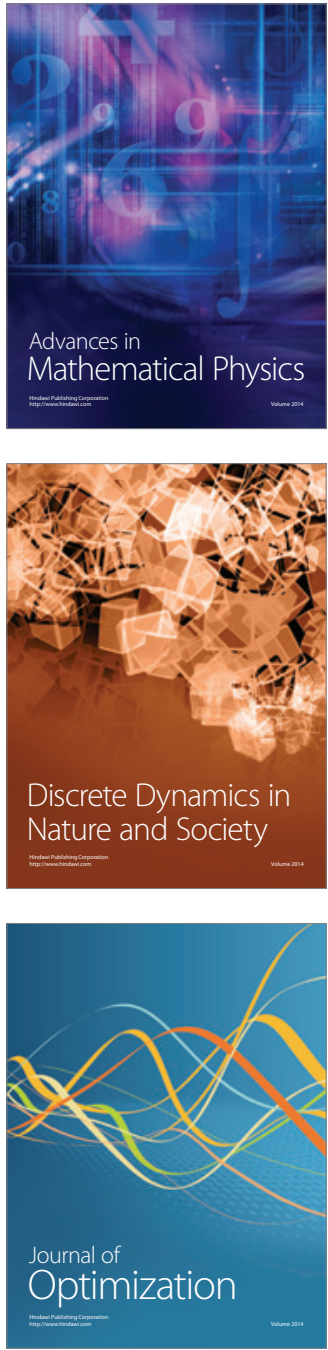